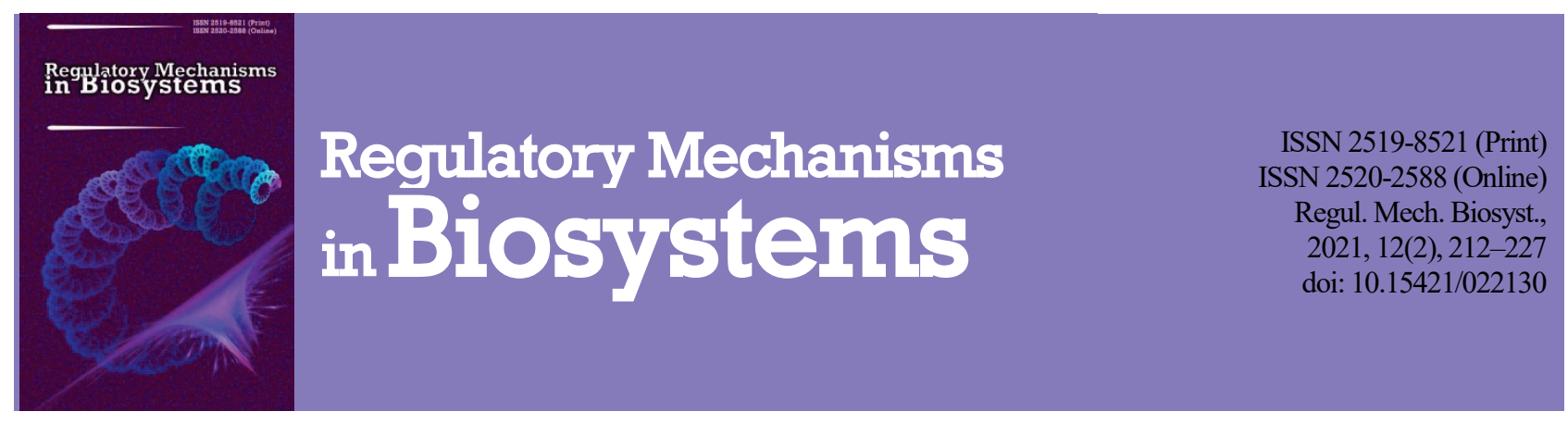

\title{
Bone remodeling stages under physiological conditions and glucocorticoid in excess: Focus on cellular and molecular mechanisms
}

\author{
V. V. Povoroznyuk*, N. V. Dedukh*, M. A. Bystrytska*, V. S. Shapovalov** \\ *D. F. Chebotarev Institute of Gerontology, National Academy of Medical Sciences of Ukraine, Kyiv, Ukraine \\ **Kyiv City Clinical Emergency Hospital, Kyiv, Ukraine
}

Article info

Received 27.03.2021

Received in revised form 02.05 .2021

Accepted 03.05.2021

D. F. Chebotarev Institute of Gerontology, National

Academy of Medical Science

of Ukraine, Vyshgorodska st.

67, Kyiv, 04114, Ukraine.

Tel.: +38-044-222-51-91.

E-mail:okfpodac@ukr.net

Kyiv City Clinical Emergency

Hospital, Bratyslavska st. 3 ,

Kyiv, 02660, Ukraine.

Tel.: + 38-044-527-69-08

E-mail:bsmp@health.kiev.ua

Povoroznyuk, V. V., Dedukh, N. V., Bystrytska, M. A., \& Shapovalov, V. S. (2021). Bone remodeling stages under physiological conditions and glucocorticoid in excess: Focus on cellular and molecular mechanisms. Regulatory Mechanisms in Biosystems, 12(2), 212-227. doi:10.15421/022130

This review provides a rationale for the cellular and molecular mechanisms of bone remodeling stages under physiological conditions and glucocorticoids (GCs) in excess. Remodeling is a synchronous process involving bone resorption and formation, proceeding through stages of: (1) resting bone, (2) activation, (3) bone resorption, (4) reversal, (5) formation, (6) termination. Bone remodeling is strictly controlled by local and systemic regulatory signaling molecules. This review presents current data on the interaction of osteoclasts, osteoblasts and osteocytes in bone remodeling and defines the role of osteoprogenitor cells located above the resorption area in the form of canopies and populating resorption cavities. The signaling pathways of proliferation, differentiation, viability, and cell death during remodeling are presented. The study of signaling pathways is critical to understanding bone remodeling under normal and pathological conditions. The main signaling pathways that control bone resorption and formation are RANK / RANKL / OPG; M-CSF - c-FMS; canonical and non-canonical signaling pathways Wnt; Notch; MARK; TGF / SMAD; ephrinB1/ephrinB2 - EphB4, TNF $\alpha$ - TNF $\beta$, and Bim Bax/Bak. Cytokines, growth factors, prostaglandins, parathyroid hormone, vitamin $\mathrm{D}$, calcitonin, and estrogens also act as regulators of bone remodeling. The role of non-encoding microRNAs and long RNAs in the process of bone cell differentiation has been established. MicroRNAs affect many target genes, have both a repressive effect on bone formation and activate osteoblast differentiation in different ways. Excess of glucocorticoids negatively affects all stages of bone remodeling, disrupts molecular signaling, induces apoptosis of osteocytes and osteoblasts in different ways, and increases the life cycle of osteoclasts. Glucocorticoids disrupt the reversal stage, which is critical for the subsequent stages of remodeling. Negative effects of GCs on signaling molecules of the canonical Wingless (WNT) $/ \beta$ catenin pathway and other signaling pathways impair osteoblastogenesis. Under the influence of excess glucocorticoids biosynthesis of biologically active growth factors is reduced, which leads to a decrease in the expression by osteoblasts of molecules that form the osteoid. Glucocorticoids stimulate the expression of mineralization inhibitor proteins, osteoid mineralization is delayed, which is accompanied by increased local matrix demineralization. Although many signaling pathways involved in bone resorption and formation have been discovered and described, the temporal and spatial mechanisms of their sequential turn-on and turn-off in cell proliferation and differentiation require additional research.

Keywords: bone cells; interactions; glucocorticoids; genomic and non-genomic effects; signaling pathways.

\section{Introduction}

The skeleton is a unique structure whose function is to maintain skeletal balance and homeostasis. In the formed skeleton, during life, old bone and damaged areas of bone are replaced with newly formed bone tissue. This cycle is called remodeling. Bone remodeling is a complex dynamic process in which local resorption and formation occurring in patterns of basic multicellular units (BMUs) in cortical and cancellous bone are coupled (Siddiqui \& Partridge, 2016; Kenkre \& Bassett, 2018). The formation of bone structures during remodeling provides a structural adaptation of bone to changes in function. During remodeling, calcium and phosphorus ions are released from bones and play an important metabolic role in mineral homeostasis.

The following cell types are involved in bone remodeling: osteoclasts, osteoblasts, including lining cells, osteomax (bone macrophages), osteocytes, and osteoprogenitor cells (Cho, 2015; Sinder et al., 2015; Novack \& Mbalaviele, 2016; Soysa \& Alles, 2019). Osteoblasts and osteoclasts, whose activities are considered in strict coupling (Chen et al., 2018; Kenkre \& Bassett, 2018), play a major role in the remodeling. Synchronous cell activity involving blood vessels contributes to the balance of resorption and bone formation in skeletal anatomical regions. However, with age, under conditions of negative factors on the body, with diseases, the remodeling cycle is disturbed; resorption begins to prevail over bone formation and can lead to osteoporosis. Osteoporosis refers to a metabolic disease in which bone mass decreases, bone microarchitecture is disrupted, and the risk of fracture increases. A severe complication in patients who take glucocorticoids is glucocorticoid-induced osteoporosis.

Glucocorticoids (GCs) have significant anti-inflammatory and immunomodulatory properties and are widely used to treat allergic, autoimmune and inflammatory diseases (Hardy et al., 2020). However, while endogenous GCs in physiological concentration support metabolic processes in bone tissue, pharmacological doses lead to loss of bone mass, development of osteoporosis and increased incidence of low-energy fractures early after oral therapy (Cherian et al., 2015). Negative changes appear both in the peripheral and axial skeleton, with a pronounced loss of bone mass in skeletal areas with a high spongy bone content, namely the vertebrae (Chuang et al., 2017). Spongy bone loss of up to $10-20 \%$ is reported in the first 6 months after therapy. Changes are also present in the compact substance of the long bones, but the rate of destruction is much lower than in the cancellous bone. In the first year of GC treatment, abnormalities in the compact bone may be only $2-3 \%$, but thereafter, this process progresses (Cherian et al., 2017). 
Glucocorticoid-induced osteoporosis (GCO) is quite common due to the large number of patients taking glucocorticoids. An estimated $1 \%$ of the US population is treated with glucocorticoids long-term (Buckley et al., 2017). Long-term glucocorticoid use leads to fractures of different localizations. The highest percentage of fractures was recorded in the vertebral bodies and in other parts of the skeleton: proximal femur, diaphyses of long bones, pelvic bones, forearm and ribs. Patients who received GC therapy for 6 months had a one-year incidence of vertebral body fractures of $5.1 \%$ and nonvertebral fractures of $2.5 \%$, and after treatment for more than 6 months, the incidence increased by $3.2 \%$ and $3.0 \%$, respectively (Amiche et al., 2016). In general, the prevalence of low-energy fractures in patients who received long-term GC treatment is high and ranges from $30 \%$ to $50 \%$ (Briot \& Roux, 2015). In the case of the same bone mineral density values, the risk of fracture in patients receiving GC therapy is significantly higher compared to patients who did not receive therapy, as well as compared to patients with postmenopausal or senile osteoporosis (Saag et al., 2016).

Despite new advances in uncovering the mechanisms of action of GCs on bone, the most frequent and severe complication is GCO (Briot \& Roux, 2015; Mazziotti et al., 2016; Cherian et al., 2017; Compston, 2018). The development of GCO is accompanied by impaired bone remodeling through increased resorption and decreased bone formation (Chotiyarnwong \& McCloskey, 2020). In these conditions, the number of remodeling units increases in the bones, many BMUs lack bone formation, which leads to bone perforation, disconnection of bone trabeculae, reduction of bone mass and bone strength.

The focus of researchers is to establish the cellular and molecular mechanisms of bone remodeling under physiological conditions and in the development of glucocorticoid-induced osteoporosis (Kameo et al., 2020; Zhang et al., 2020) identifying the group of patients with impaired remodeling and high risk of fracture are key to preventing and developing treatment tactics for this severe complication (Xu et al., 2019).

Multiple signaling pathways that regulate bone remodeling have been identified, but the question of the molecular mechanisms of disruption, requires further study. Multiple molecules and signaling pathways are active at each stage of remodeling, affecting differentiation, activation, and viability of osteoblasts and osteoclasts (Deng et al., 2019; Zhao et al., 2020a, b). Information about the effects of glucocorticoids on bone remodeling stages is fragmentary, further studies on the effects on signaling pathways and cells at different stages of remodeling are needed.

Purpose of the review: to analyze and summarize the information on the cellular and molecular mechanisms acting at different stages of bone remodeling in physiological conditions and after exposure to glucocorticoids in excess on the basis of literature data.

\section{Effect of excess glucocorticoids on bone}

The action of GCs on inflammation, organs and tissues of the body, including bone tissue, has genomic and non-genomic effects. The genomic action of GCs is carried out after GC binding in cell cytoplasm to its receptor (GR), with subsequent translocation of the complex into nucleus, action on DNA with regulation of genes. In the mechanism of nongenomic action of GCs, four directions were identified and summarized: membrane-bound GR (mGR) actions, cytosolic GR actions, direct physicochemical interaction of GCs with the cellular membranes, and mitochondrial GR signal transduction (Hartmann et al., 2016; Panettieri et al., 2019). Glucocorticoids are lipophilic molecules that diffuse across the cell membrane into the cytoplasm. In the cytoplasm, GR is bound to immunophilin ligands (Cyp 55, PP5, FKBP51, and FK50652), heat shock proteins (hsp70, hsp90, and hsp23), which function as molecular chaperones that increase the GC ligand affinity and provide GR resistance to ubiquitination and proteosomal degradation (Hartmann et al., 2016). After remodeling of the complex, GR binds to the GC and moves to the nucleus (Fig. 1).

The GR is expressed in osteoblast precursor cells, osteoblasts, osteocytes, and osteoclasts (Bouvard et al., 2009; La Corte et al., 2010). Human (h) GR is the product of a gene located in chromosome 5q31-32 consisting of 10 exons. The hGR has been found to have 3 different functional domains (Oakley \& Cidlowski, 2013). The N-terminal immunogenic domain, which contains most of the residues subject to posttranslational modifications, has a transcriptional activation function (AF-1); the central, DNA-binding domain consists of two zinc fingers implicated in DNA binding, translocation into the nucleus, and GC dimerization; the hinge region binds the central DNA-binding domain and the ligand-binding domain; the C-terminal ligand-binding domain (AF-2) interacts with ligand-dependent coregulators (Nicolaides et al., 2010; Oakley \& Cidlowski, 2013; Cruz-Topete \& Cidlowski, 2015).

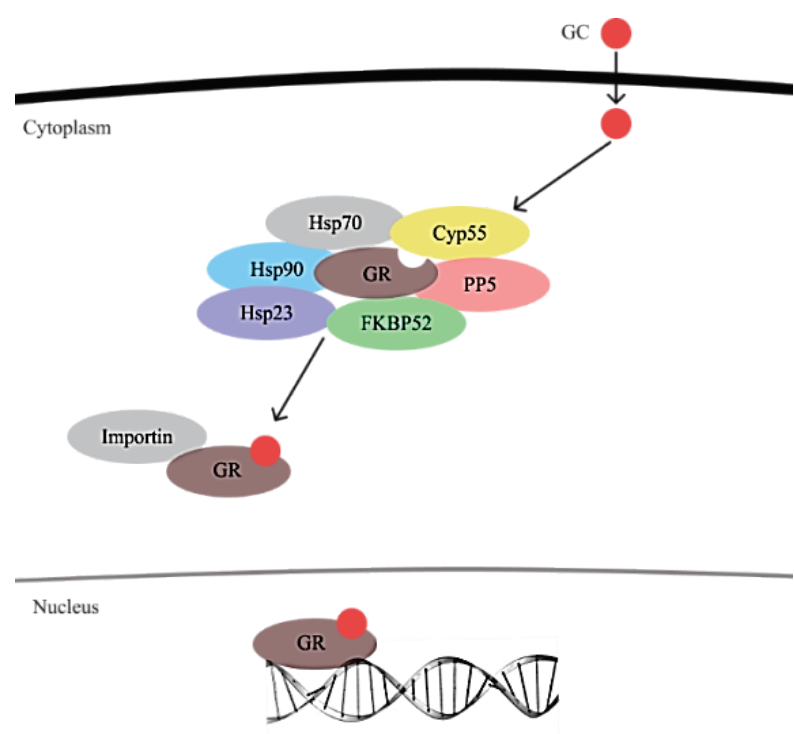

Fig. 1. Association of the glucocorticoid receptor with the glucocorticoid (adapted Todosenko et al., 2017): GC - glucocorticoid; GR - glucocorticoid receptor; Cyp 55, PP5, FKBP51, and FK50652 - immunophilins; hsp70, hsp90, and hsp23 - heat shock proteins

There are GR protein isoforms (namely, GR $\alpha$, GR $\beta$, GR $\gamma$, GR-A, and GR-P) generated from the same GR gene through alternative splicing and use of alternative translation initiation sites (Smith \& Cidlowski, 2010). The most widely expressed isoforms of hGR $\alpha$ (h-human) and hGR $\beta$. As a multiprotein complex, hGR $\alpha$ is present in the cytoplasm of almost all human cells (Kelly et al., 2008). After binding GC to GR, the complex enters the nucleus, where it functions as a ligand-dependent transactivation and transrepression factor directly affecting DNA. hGR $\beta$ is mainly located in the nucleus, does not bind glucocorticoids, is transcriptionally inactive, and regulates the expression of numerous of genes independent of GR $\alpha$ activity (Hartmann et al., 2016; Todosenko et al., 2017). GR $\beta$ acts as a repressor of cytokine gene transcription through recruitment of histone deacetylase complexes.

To prove the direct effect of glucocorticoids on bone, 50 healthy volunteers of postmenopausal women receiving $5 \mathrm{mg}$ of prednisolone were studied to exclude the action of inflammatory mediators that are expressed in patients with pathology (Ton et al., 2005). The study was carried out at 2, 4, 6 and 8 weeks. There were revealed decreased indices of osteoblast activity in serum: procollagen type I N-terminal propeptide (PINP), procollagen type I carboxy-terminal propeptide (PICP), osteocalcin and bone specific alkaline phosphatase (BSALP) as well as indices of osteoclast activity: serum cross-linked N-telopeptides of type 1 collagen (NTX) and free urinary deoxypyridinoline (DPD). It was found that $5 \mathrm{mg}$ of prednisolone in healthy women suppresses bone formation, which can adversely affect bone strength. This study proved that prednisolone affects bone regardless of inflammation. Inflammatory mediators and other deleterious molecular factors that are expressed in inflammatory diseases, with the summation of the negative effects of GC therapy, lead to poor bone quality (Ton et al., 2005).

Modulation of GC action can be carried out at the tissue level by various mechanisms: variations in the expression and sensitivity of receptors, transmembrane transporters, by enzymatic metabolism. Glucocorticoids modulate the expression of $11 \beta$-hydroxysteroid dehydrogenase type 1 and

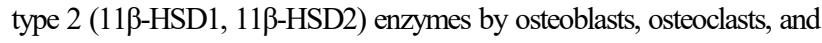
osteocytes (Hachemi et al., 2018). These enzymes affect the differentiation 
and function of bone cells. The most active enzyme is 11ß-HSD1, a glucocorticoid action receptor-modulator, one of the functions of which is the conversion of active GCs cortisol and corticosterone into inactive analogues of cortisone and dehydrocorticosterone. It is this enzyme that plays a crucial role in the development of GCO, mediating the harmful effects of GCs on bone cells and bone formation (Fenton et al., 2019). The biosynthesis of this enzyme increases with age and in GCO, which is accompanied by impaired bone quality and an increased risk of fracture. In addition, GCs block the expression of the enzyme 11/-HSD2, which protects osteoblasts and osteocytes from apoptosis. Decreased expression of this enzyme is recorded with age, further enhancing the negative effects of

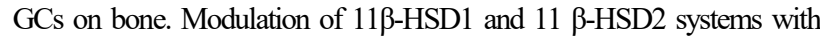
age and biosynthesis of proinflammatory cytokines may be key mechanisms of bone response to $\mathrm{GCs}$ action and requires further research (Cooper et al., 2003; Hardy et al., 2018).

The active form of vitamin $\mathrm{D} 1,25(\mathrm{OH}) 2 \mathrm{D} 3$, which is formed in the kidneys, plays a central role in calcium homeostasis, bone metabolism, and bone remodeling. Experimental studies have confirmed the concept that there is a local regulation of vitamin D metabolism in osteoblasts (Siddiqui \& Partridge, 2016). Human osteoblasts express vitamin D receptor (VDR) mRNA and they also activate and deactivate enzymes involved in vitamin D conversion (Zaynya et al., 2019). Key enzymes in vitamin D metabolism are members of the cytochrome P450 superfamily (CYP27A1 (as vitamin D 25-hydroxylase), CYP27B1, and CYP24A1). The effects of vitamin D $(25(\mathrm{OH}) \mathrm{D} 3$ and $1,25(\mathrm{OH}) 2 \mathrm{D} 3$ on CYP27B1 and CYP24A1 enzymes in bone cells have been studied (Wegler et al., 2016). In osteoblast culture, $25(\mathrm{OH}) \mathrm{D} 3$ was shown to be converted to the active form $1,25(\mathrm{OH}) 2 \mathrm{D} 3$ under the action of the CYP27B1 enzyme, which stimulates osteoblast proliferation and maturation and increases intercellular mineralization (Pathak et al., 2020). In vitro studies have proven that not only osteoblasts but also osteocytes and osteoclasts have VDR, express CYP27B1, can locally convert vitamin D to the active metabolite $1,25(\mathrm{OH}) 2 \mathrm{D} 3$, and by expressing the enzyme CYP24A1 can locally catabolize vitamin D (Zarei et al., 2016).

Based on their own data, the authors assumed the existence of a previously unknown mechanism of the effect of GCs on human bone metabolism through cytochrome P450 superfamily enzymes in bone cells (Wegler et al., 2016). The obtained data reveal new possibilities of vitamin D influence on metabolic processes in bone cells. It is suggested that one of the mechanisms by which glucocorticoids have a negative effect on bone may be the disruption of vitamin D conversion in osteoblasts (Wegler et al., 2016).

There are negative effects of excess GCs on bones, disruption of GCs function of organs and systems - a combined pathway to the development of osteoporosis (Panettieri et al., 2019). Glucocorticoids negatively affect the expression and metabolism of growth hormone and thyroid hormones, insulin-like growth factor 1 (IGF-1), and insulin-like growth factor-binding protein-1 (IGFBP-1) (Mazziotti et al., 2016). Excess GCs inhibit sex hormone synthesis both indirectly by reducing pituitary hormone levels and adrenal androgen production, and directly by disrupting hormone production by the gonads (Cherian et al., 2017). Under the influence of glucocorticoids, the secretion of luteinizing hormone is reduced. This leads to a decrease in estrogen synthesis by the ovaries, the deficiency of which plays a significant role in the pathogenesis of GIO. The suppression of sex hormone biosynthesis caused by excess glucocorticoids leads to muscle weakness, which is a risk factor for falls and fractures (Briot \& Roux, 2015; Cherian et al., 2017).

Under the influence of excess GC in the intestine, absorption of calcium and phosphorus is reduced. This is associated with impaired functionality of the calcitriol receptor $(1,25(\mathrm{OH}) 2 \mathrm{D} 3)$ in the intestinal mucosa, which leads to decreased biosynthesis of calcium-binding protein (calmodulin). The level of calcium excretion is increased, primarily due to the direct effect of GCs on tubular reabsorption.

Decreased intestinal calcium absorption and increased renal excretion leads to increased serum PTH levels, development of secondary hyperparathyroidism, and increased bone resorption.

In recent years, a new mechanism of osteoporosis development has been described as autophagy (cell recycling process). Autophagy is important for regulating bone remodeling and maintaining bone homeostasis
(Shapiro et al., 2014; Shen et al., 2018; Wang et al., 2019a). Regarding the effect of GCs on bone cell autophagy, our knowledge is limited and the available data are contradictory. It has been reported that in glucocorticoidinduced osteoporosis, autophagy inactivation does not affect the number of osteoblasts or the expression of osteoblast-related genes such as osteocalcin, collagen type I alpha $1(\mathrm{Col} / \alpha 1)$ and runt-related transcription factor 2 (RUNX2) (Lin et al., 2016). However, another study has shown that during chronic glucocorticoid therapy, suppression of autophagy in osteoblasts increases their apoptosis (Wang et al., 2019a), promotes progression of GC-induced osteoporosis, and increases the risk of low-energy fractures (Han et al., 2018). When considering autophagy and its role in osteoblast function, at least two factors must be considered: the crosslinking between autophagy and apoptosis, and the GC dose (Wang et al., 2019b). The above-mentioned changes in bone tissue under the influence of GCs in excess, supplemented by the data below in the text, are reflected in bone remodeling.

\section{Physiological bone remodeling cycle}

The bone remodeling cycle is usually considered to involve four stages: activation, resorption, reversal and formation (Feng \& McDonald, 2011) or additionally two more stages are distinguished, namely the resting stage (resting bone) preceding the activation stage and the terminal stage (Siddiqui \& Partridge, 2016) (Fig. 2).

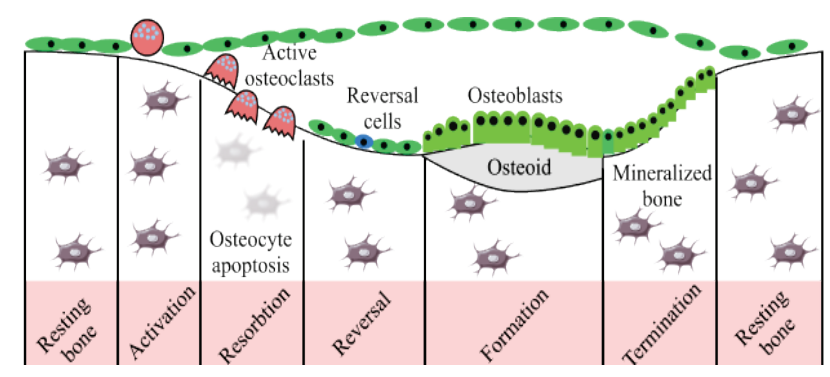

Fig. 2. Schematic representation of a basic multicellular unit (BMU) of cancellous bone. Stages of remodeling (explanation in text) (adapted from Kenkre \& Bassett, 2018)

According to Parfitt (1980), resorption in the BMU of adult bone takes about 3 weeks and bone formation takes $3-4$ months. Overall, about $5-10 \%$ of the skeleton is replaced each year, and the entire adult skeleton is renewed within 10 years. The following cell types are involved in bone remodeling: osteoclasts, osteoblasts, bone lining cells, osteomax (bone macrophages), osteocytes and osteoprogenitor cells. Synchronous activity of the cells with the participation of blood vessels contributes to the balance of resorption and bone formation in the anatomical areas of the skeleton.

Various local and systemic factors control the bone remodeling cycle, some of which have been identified. The most important mechanism of remodeling of bone at all stages is the close molecular communication between osteoblasts, osteoclasts and osteocytes, which is provided by many cytokines, hormones and signaling pathways.

Resting stage (resting bone). The trabecular surface is covered bone lining cells, which have a flattened shape and low metabolic rate. Their function is not fully understood (Florencio-Silva et al., 2015). Bone lining cells differ from osteoblasts in phenotype. At this stage, the cells prevent direct interaction between the osteoclast and the matrix.

Activation stage. The activation stage can be considered as a complex of signals affecting the bone and initiating the BMU formation site. A prerequisite for remodeling is the death of osteocytes in the local area, occurring in various ways. This can be mechanical loading associated with deformation, pressure disturbance in the osteocyte lacuno-canalicular system, structural damage to the bone area, bone marrow condition, homeostasis changes, and other factors that activate osteocyte apoptosis.

Some key biomarkers were identified and their potential role in the mechanisms of osteocyte proapoptotic signal transduction leading to osteocyte death by various pathways was established. Among them: ROS, BCL-2-associated X protein (BAX), the member of the BCL-2 family 
(PUMA), homocysteine, the high-mobility group box 1 (HMGB1), Fasassociated protein with death domain (FADD), sclerostin, adenovirus E1B $19 \mathrm{kDa}$ interacting protein 3 (BNIP3), connective tissue growth factor (CTGF, known as CCN2), TNF- $\alpha$, caspase-3, cathepsin K, DMP-1, proline-rich tyrosine kinase 2 (Pyk2) (Ru \& Wang, 2020).

Resorption stage. Osteoclasts are key players in remodeling bone, skeletal health and disease (Kim \& Kim, 2016; Novack \& Mbalaviele, 2016). Osteoclasts attach to bone at sites of osteocyte death. The resorption is a multistep process consisting of several sub-stages - expression of biologically active molecules by osteoblasts and osteocytes, formation of osteoclast precursor cells, their fusion and osteoclast maturation. The key role is assigned to osteoblasts, but an inverse relationship, the influence of osteoclasts on the maturation and activity of osteoblasts was also revealed (Matsuoka et al., 2014).

An osteoclast is formed from a hematopoietic stem cell. In the early stage of osteoclast differentiation, signaling through macrophage colony stimulating factor (M-CSF) and its macrophage colony stimulating factor receptor (c-FMS) plays a significant role. M-CSF is expressed by mesenchymal stromal cells, cells lining cells, osteoblasts and endothelial cells. Transcription factor PU.1 induces cytokine M-CSF binding to the cellsurface receptor c-FMS, involving micropthalmia-associated transcription factor (MITF) and CCAAT-enhancer binding protein $\alpha(\mathrm{C} / \mathrm{EBP} \alpha)$ proteins. In committed cell formation, the M-CSF - c-FMS complex activates the cellular oncogene Fos (c-FOS) / nuclear factor kappaB (NF-kB) / nuclear factor of activated T cells (NFATc1) signaling pathway (Huntley et al., 2019).

Many cytokines are involved in osteoclastogenesis, the key ones being M-CSF and RANKL. M-CSF expression is induced by TNF- $\alpha$, IL$1 \beta$, IL-6, IL-7, and IL-15; RANKL expression is upregulated by PTH, $1,25(\mathrm{OH}) 2 \mathrm{D} 3$, the proinflammatory cytokines IL-1, IL- 6 , IL-17, and TNF- $\alpha$ (Braun \& Zwerina, 2011; Jann et al., 2020). To date, the ideas about the role of cytokines in osteoclastogenesis have expanded. Cytokines that promote osteoclastogenesis have been identified: TNF- $\alpha$, IL-1, IL-6, IL-7, IL-8, IL-11, IL-15, IL-17, IL-23, and IL-34, also anti-osteoclastogenic cytokines including interferons (IFN- $\alpha$, IFN- $\beta$, IFN- $\gamma$ ), IL-3, IL-4, IL-10, IL-12, IL-27, and IL-33 (Amarasekara et al., 2018). IFN- $\alpha$ and IFN- $\beta$ reduce osteoclastogenesis by inhibiting RANKL expression by osteoblasts. TNF- $\alpha$ has a strong synergistic effect on osteoclast differentiation. In stromal cells, TNF- $\alpha$ can induce osteoclastogenesis in the presence of sufficient levels of RANKL and M-CSF (Kitaura et al., 2013). Osteoclastogenesis is regulated by IL-6, via cytosolic $\mathrm{Ca}^{2+}$ sensitive levels. High $\mathrm{Ca}^{2+}$ levels lead to inhibition of IL- 6 , which prevents bone resorption.

RANKL are expressed by osteoblasts, bone lining cells, osteoprogenitor cells and osteocytes. There is evidence that compared to osteoblasts, osteocytes express more RANKL and have a greater ability to support osteoclastogenesis than osteoblasts or bone marrow stromal cells (Nakashima et al., 2011). RANKL is also expressed by immunocompetent cells located in the bone marrow, activated T-lymphocytes, B-lymphocytes, monocytes, macrophages, etc. The RANKL protein recognizes and binds to the RANK receptor located on the plasma membrane of osteoclast line cells and promotes their differentiation and activation. The RANKL RANK connection is considered to be crucial in the activation of osteoclastogenesis.

Blocking of RANKL to RANK is performed by OPG, which is accompanied by a decrease in differentiation and activity of osteoclasts. Osteoprotogerin is expressed by osteoblasts, lining cells and osteocytes, as well as B-lymphocytes located in bone marrow, which account for $64 \%$ of total OPG biosynthesis (Li et al., 2007). That is, RANKL / RANK / OPG form an important system that regulates a biological process such as osteoclast differentiation, activation or inhibition. Additionally, the leucinerich repeat-containing $\mathrm{G}$ protein-coupled receptor 4 (LGR4) expressed by osteoclasts may be acted on as another decoy receptor for RANKL (Chen et al., 2018).

In response to signals received by the cell through the RANKL RANK link and involvement of the tumour necrosis factor receptor-associated factor (TRAF6) adaptor molecule, three signaling pathways are activated, closing in on NFATc1. The c-Jun N-terminal kinase (JNK) signaling pathway mobilizes osteoclastic transcription factors associated with c-Fos antigens (Fra-1 and 2) / NFATc1, another pathway is extracellular signal-regulated kinase 1 (ERK1) / NF-kB / activator protein 1 (AP-1) / MITF and p38 / AP-1 / MITF signaling (Fig. 3) (Boyce \& Xing, 2008; Kanakamedala et al., 2019; Soysa \& Alles, 2019).

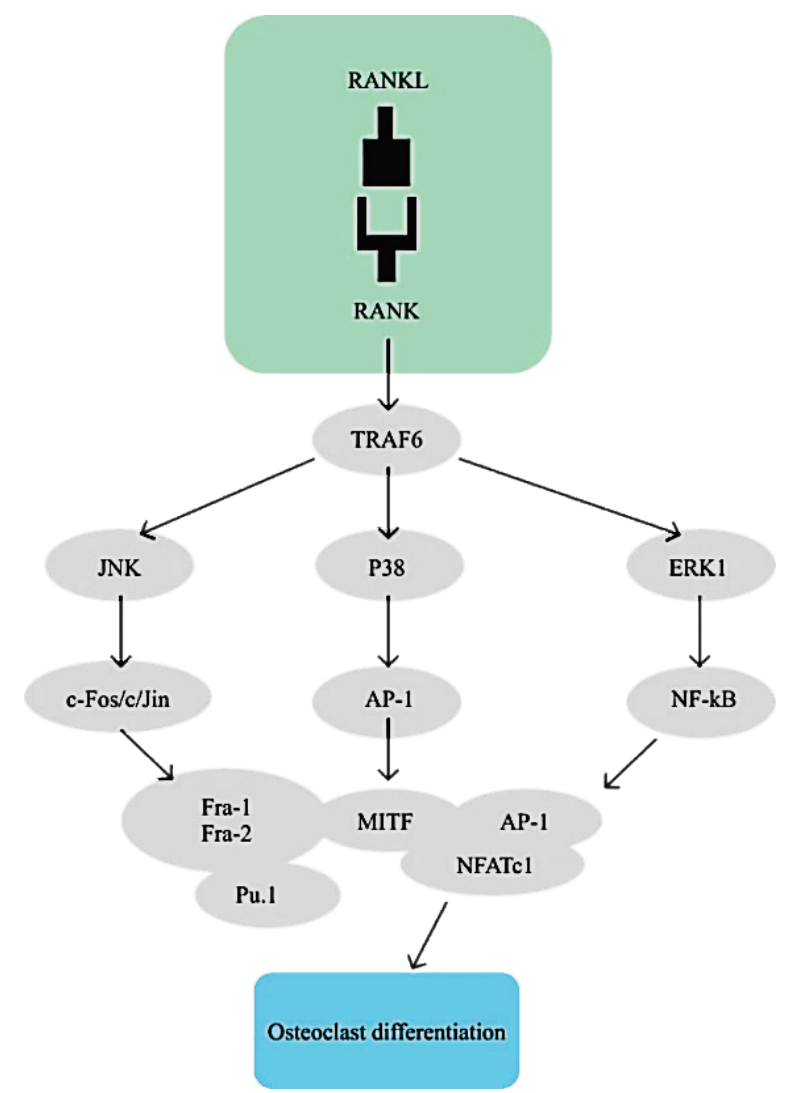

Fig. 3. Signaling pathways activated by the RANKL - RANK complex in osteoclast differentiation: AP-1 - Activator protein 1; c-Fos, Fra-1 and Fra-2 - Member of the Fos transcription factor family; JNK - c-Jun Nterminal kinase; MITF - Micropthalmia-associated transcription factor; NFAT $\mathrm{c} 1$ - Nuclear T cell activator factor c-1; NF-kB - Nuclear factor kappa B; PU.1 - transcription factor; RANK - receptor activator of NFkappa B; RANKL - receptor activator of NF-kappa B ligand; TRAF6-

Tumor necrosis factor receptor-associated factor

M-CSF - c-FMS and RANKL - RANK complexes induce expression of osteoclast-specific markers via NFATc1 such as dendritic cell-specific transmembrane protein (DC-STAMP), osteoclast stimulatory transmembrane protein (OC-STAMP), small mothers against decapentaplegic (SMAD1/5/9), tartrate-resistant acid phosphatase (TRAP), v-ATPase subunit $\mathrm{d} 2$ (Atp6v0d2), OC-associated receptor (OSCAR), the tyrosine kinases of the Src family (SRC), $\beta 3$-integrin, osteopetrosis-associated transmembrane protein 1 (OSTM1), B-lymphocyte induced maturation protein and cathepsin K, and matrix metallopeptidase 9 (MMP-9) (Boyle et al., 2003; Cheon et al., 2016; Amarasekara et al., 2018; Huntley et al., 2019; Fu \& Shi, 2020; Kameo et al., 2020; Søe, 2020).

The c-Jun N-terminal kinase (JNK), p38, and ERK signaling pathways that induce osteoclast differentiation are activated in response to signals received by the cell through RANKL binding to the RANK receptor and involvement of the adaptor molecule tumour necrosis factor receptor-associated factor 6 (TRAF6). The JNK signaling pathway mobilizes osteoclast transcription factors associated with c-Fos antigens (Fra-1 and Fra-2) and NFATc1. Other pathways that also loop to NFATc1 are ERK1 / NF-kB / activator protein 1 (AP-1) / MITF and ERK1 / NF-kB / AP-1 (Boyce \& Xing, 2008; Soysa \& Alles, 2019).

NFAT (NFATc1-4) belongs to the family of transcription calciumdependent factors, which function to coordinate cell growth and differentiation by regulating the production of key molecules of these processes: growth factors, various cellular proteins and cytokines (Mandal et al., 2016). NFAT5, a member of this family, is a calcium-independent factor. In resting cells, NFATs are hyperphosphorylated and in an inactive state. Osteoblast and osteoclasts' differentiation and activity is associated with 
calcium signaling. An increase in calcium levels in osteoblasts involving bone morphogenetic protein (BMP)-2 activates calcineurin, which dephosphorylates NFATc1, facilitating its import into the nucleus (NegishiKoga \& Takayanagi, 2009). In the nucleus, NFATc1 interacts with active transcription factors such as osterix, AP-1, Pu.1, Fra and, after binding to DNA sites, activates transcription. NFATcl is important for the regulation of osteoblast differentiation and osteoblast-mediated osteoclast activity (Mandal et al., 2016).

Crossed with the RANKL - RANK signaling pathway is the BMP signaling pathway (Fig. 4) via the mitogen-activated protein kinase kinase kinase binding protein (MAP3K), activated by proteins of fusion TAK1TAB1 (Qi et al., 2014; Huntley et al., 2019). The BMPR1/BMPR2 complex affects osteoclast differentiation through activation of canonical signaling pathway proteins: SMAD1/ 5, SMAD4, ERK, and NF-kB (Broege et al., 2013; Fong et al., 2013; Huntley et al., 2019). Differentiation of macrophages into osteoclasts and activity of mature osteoclasts is enhanced with a decrease or loss of Smad1 / 5 expression (Tasca et al., 2018).

Secreted members of the BMP family, BMP-2 and BMP-7, are involved in comminuted cell fusion and osteoclast formation through Smad1 / 5/9 activation (Omi, 2019). BMP transduce intracellular signaling via the canonical SMAD signaling pathway and non-canonical SMAD signaling pathways such as mitogen-activated protein kinases (MAPK) / phosphoinositide 3-kinase (PI3K) / AKT. Differentiation of macrophages into osteoclasts and activity of mature osteoclasts is enhanced with decreased or loss of Smad1/ 5 expression (Tasca et al., 2018).

Vitamin D metabolites can inhibit the fusion of osteoclast precursors by reducing the expression of NFATcl and DC-STAMP (Zarei et al., 2016). In addition, smaller osteoclasts are formed with fewer nuclei per osteoclast.

The formation of osteoclasts also involves the Notch signaling pathway, which transmits signals between cells through proteins of the Notch family and involves a large number of different genes, including genes responsible for a variety of cellular functions, including differentiation and proliferation (Zanotti \& Canalis, 2016). In osteoclast precursor cells, stimulation of Notch signaling leads to the formation of large osteoclasts with numerous nuclei and increased resorption activity, but the resorptive activity of small osteoclasts is suppressed.

In addition to the previously described pathways, other pathways in the regulation of osteoclastogenesis are being investigated. A new mechanism of osteoclast differentiation has been described and the role of protein kinase D from the kinase family in the regulation of osteoclast activity has been established (Leightner et al., 2020).

PTH has a mediated effect on osteoclast differentiation via the receptor on osteoblasts PTH1R. This link leads to activation of RANKL expression by osteoblasts. In addition, PTH binding to PTH1R on osteo- clasts promotes V-ATPase activity by inducing expression of V-ATPase a3-subunit and d2-subunit, enhances its resorptive properties (Liu et al., 2016). The association of PTH with PTH1R in osteoclasts inhibits the expression of SEMA4, which is accompanied by a decrease in osteoblast activity.

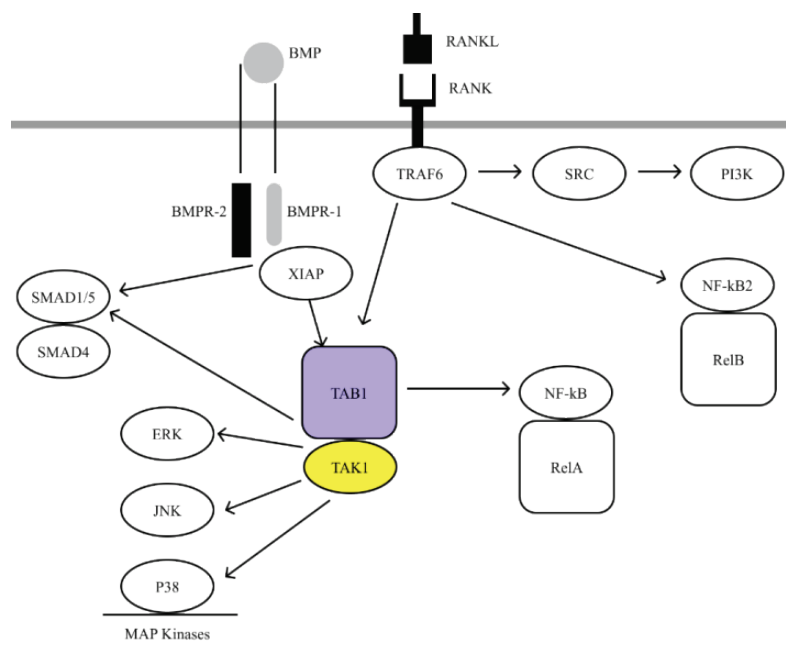

Fig. 4. BMP and RANKL signaling pathways: cross-activation of MAP kinases (adapted from Huntley et al., 2019); BMP - bone morphogenic protein; BMPR1, 2 - receptors BMP; ERK - extracellular-signalregulated kinase; JNK - c-Jun N-terminal kinase; NF-kB - nucleus factor kappa B; PI3K - phosphoinositide 3-kinase; RelA (p65), RelB (C1E4) NF-kB family members; SMAD - small mothers against decapentaplegic, SRC - family of protein tyrosine kinases; TRAF - tumour necrosis factor receptor-associated factor; XIAP - X-linked inhibitor of apoptosis, TAK1-TAB1 - fusion proteins

Fifteen new genes involved in RANKL-mediated signal transduction for osteoclastogenesis were identified, including Merlot, Tussilagone, and Lrp1 (Yamakawa et al., 2020). The Merlot gene simultaneously inhibits osteoclastogenesis through the GSK $3 \beta$ - NFATc1 axis and induces apoptosis, reducing the lifespan of osteoclasts.

Osteoclast viability is maintained by M-CSF and RANKL (Fig. 5). After M-CSF binds to its c-FMS cognate receptor, two signaling pathways are activated - MARK (Ras / Raf / MEK / ERK) and PI3K / AKT kinase / mammalian target of rapamycin (mTOR) pathways, the latter being also synergistically activated by RANKL - RANK complex (Soysa \& Alles, 2019).

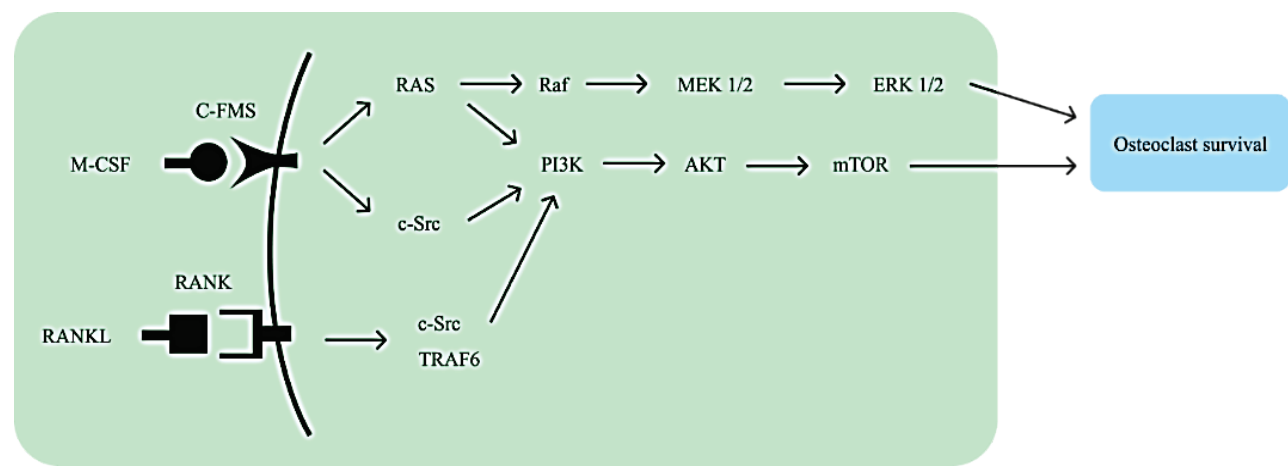

Fig. 5. MARK signaling pathway activated by M-CSF - c-FMS; PI3K / AKT / mTOR signaling pathway simultaneously activated by M-CSF - c-FMS and RANKL - RANK in the maintenance of osteoclast viability (adapted from Soysa \& Alles, 2019): AKT - kinase, ERK - extracellular signalregulated kinase, MEK - kinase, mTOR - mammalian target of rapamycin, PI3K - phosphoinositide 3-kinase, Src - family of protein tyrosine kinases, TRAF6 - tumour necrosis factor receptor-associated factor 6, TNF6 - tumor necrosis factor 6

The M-CSF - c-FMS complex also activates the Ras/Raf/PAK pathway, which leads to increased osteoclast viability, by modulating the expression of Survivin, a protein member of the apoptosis protein inhibitor (IAP) family (Soysa \& Alles, 2019). Activated osteoclasts attach to the bone surface via integrins ( $\alpha \mathrm{V} \beta 3$ and others) with the formation of the podosome and subsequent formation of a membrane ruffling (Fig. 6) (Feng \& Teitelbaum, 2013; Teitelbaum, 2015). In addition to adhesion, integrin $\alpha v \beta 3$ is involved in osteoclast migration (Mellis et al., 2011; Kanakamedala et al., 2019). The formation and function of actin that makes up the osteoclast cytoskeleton is regulated by Rho family proteins (GTPases). Ras 
homolog family member $\mathrm{A}$ (RhoA) proteins are involved in the regulation of actin cytoskeleton of osteoclast: cell division cycle 42 (Cdc42) regulates filopodia formation, Rac protein (subfamily of the Rho family of GTPases), regulates the formation of a membrane ruffling, and RhoA protein increases adhesion and tension of actin fibers (Morel et al., 2018).

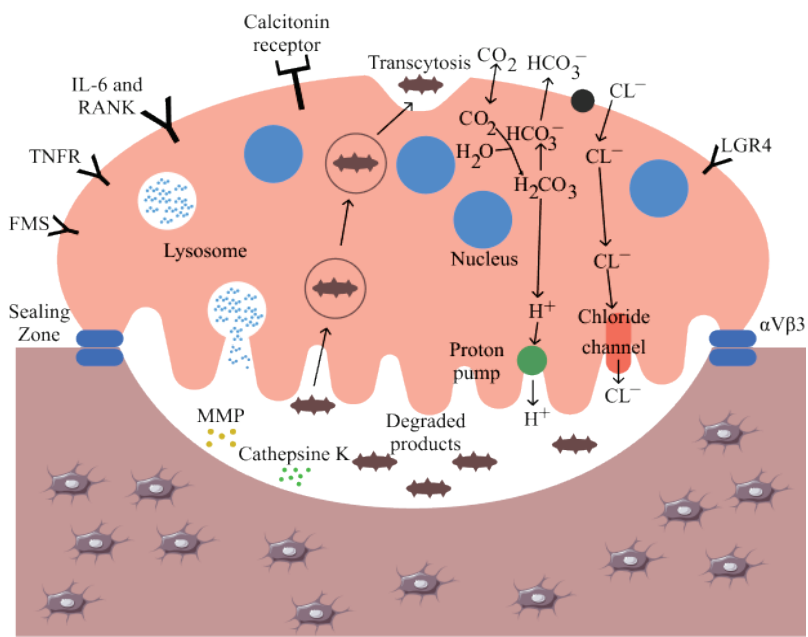

Fig. 6. Schematic representation of active osteoclast: activated osteoclasts attach to the bone surface through integrins, which bind to bone vitronectin, forming an edge seal around the resorption area; through the formed ruffled border of the membrane, hydrogen ions enter the tight seal through a proton pump, chlorine ions through chloride channels, proteases and acid phosphatases are released from lysosomes, which leads to the destruction of the matrix; osteoclasts are characterized by transcytosis; residues of detritus after degradation in lysosomes are removed from the cell and enter the bloodstream; on the opposite surface of the osteoclast there are numerous receptors that receive regulatory signals: FMS, RANK and interleukin-6, TNFR, LGR4, calcitonin receptor, etc.)

Resorption is accompanied by the formation of a lacuna approximately $60 \mu \mathrm{m}$ deep in the bone (Boyce et al., 2018). To resorb the mineral part of bone, osteoclasts produce hydrochloric acid, releasing $\mathrm{H}^{+}$ions through the proton pumps of the cell membrane and Cl-ions through the chloride channels. To destroy the collagen matrix, osteoclasts produce matrix metalloproteinases and cathepsin (Feng \& Teitelbaum, 2013).

Bone resorption by osteoclasts is controlled by four major hormones (calcitonin, PTH, vitamin D and estrogens), many signaling pathways and biomarkers. At this stage, the expression of RANKL, M-CSF increases, granulocyte macrophage colony-stimulating factor (GM-CSF), dendritic cell-specific transmembrane protein (DC-STAMP), integrin $\alpha v \beta 3$, retinoic acid, sphingosine-1-phosphate (S1P), PTH, relaxin. Bone resorption by osteoclasts is also activated by Wnt5a - receptor tyrosine kinase-like orphan receptor 2 (Ror2) signaling through activation of dishevelled-associated activator of morphogenesis 2 (Daam2) / Rho / protein kinase N3 (Pkn3) / c-Src pathways (Uehara et al., 2019). Osteoclast activity is supported by Sema $3 \mathrm{~A}$.

OPG, IL-4, IL-18, estrogen, calcitonin, and transforming growth factor-beta (TGF- $\beta$ ) act as resorption inhibitors. Estrogens via receptor $\alpha$ and $\beta$ expressed by osteoclasts, by inhibiting RANKL synthesis by osteoblasts and osteocytes, reduce osteoclast formation, stimulate OPG expression, and suppress osteoclast formation by reducing IL-1, IL-6, IL-11, TNF- $\alpha$, TNF- $\beta$ and M-CSF levels (Florencio-Silva et al., 2015). Calcitonin receptor interaction with calcitonin suppresses preosteoclast fusion and causes loss of the ruffled border osteoclast, resulting in inhibition of bone resorption (Xie et al., 2020).

Reversal stage. The transition to the formation stage occurs through the reversal stage, which includes apoptosis of osteoclasts, preparation of the surface for settlement by osteoblasts, formation of canopies over the resorption area, proliferation and differentiation of osteoblasts. The signaling pathways for switching resorption to the reversal stage are not fully understood.

There are two points of view on the nature of the cells located in the BMU immediately after resorption by osteoclasts, which are involved in preparing the resorbed surface for settlement by osteoblasts. Based on immunohistochemical examination, $97 \%$ of the cells were positive for the osteoblast marker RUNX2. They were located adjacent to osteoclasts, which, according to the authors, indicates colonization of the cavity by osteoblasts immediately after resorption (Andersen et al., 2013). According to other authors, the resorption cavity initially contains bone macrophages or osteomax with a characteristic phenotype and the presence of F4/80 marker (Wu et al., 2013; Cho, 2015; Sinder et al., 2015). The function of cells in the reversal stage is to remove residual mineralized and non-mineralized matrix (detritus) by phagocytosis and prepare the surface of bone for settlement by osteoblasts (Jensen et al., 2011; Sinder, 2015; Kenkre \& Bassett, 2018). Bone lining cells produce collagen, which is deposited as a thin layer on the surface of BMU (Matsuo \& Irie, 2008).

A feature of this stage is the location above the BMU region of the canopies (Fig. 2), which are a reservoir of osteoprogenitor cells (Kenkre \& Bassett, 2018). These cells have a flattened appearance, they are similar to osteoblasts, can proliferate and renew. A significant role in the formation of canopies is played by bone lining cells. Canopies close the bone remodeling BMU compartment, which separates osteoclasts and osteoblasts from bone marrow (Pettita et al., 2008). Activating factors for the formation of osteoprogenitor canopies are: TGF- $\beta$, IGF-1, IGF-2, BMPs, PDGF, fibroblast growth factor (FGF), and estrogens (Siddiqui \& Partridge, 2016). The canopies of osteoprogenitor cells are permeated with capillaries that provide rapid access to systemic regulatory factors to control osteoclasts and osteoblast precursors that migrate to this area (Andersen, 2009). Osteoprogenitor cells differentiate into osteoblasts and also participate in the settlement of the surface resorbed by osteoclasts.

Formation stage. The main events of this stage are the formation of osteoblasts from mesenchymal stem cells in the resorption cavities, their proliferation, differentiation and mineralization of the osteoid. At this stage joint action (coupling) of osteoclasts and osteoblasts is clearly manifested. In recent years, a number of important regulatory transcription factors contributing to bone formation and mineralization have been identified.

Osteoclasts play an important role in the transition to the reversion and formation stage (Wang et al., 2020). One of the ways is bidirectional stimulation of osteoblastogenesis or inhibition of osteoclastogenesis through ephrins (Matsuo \& Otaki, 2012; Plotkin et al., 2019). Osteoblasts express ephrin $\mathrm{A}$ and $\mathrm{B}$ ligands as well as their $\mathrm{Eph} A$ and $\mathrm{EphB}$ receptors, whereas osteoclasts express only ephrin A2, B1, and B2 ligands (Plotkin et al., 2019). Stimulation of osteoblast proliferation through the ephrinB1/ ephrinB2 - EphB4 signaling pathway is carried out through the transcription factors RhoA, RUNX2, PDZ-binding motif (TAZ). Inhibition of osteoclast function is carried out through the c-Fos - NFAT signaling pathway (Fig. 7). These signaling pathways confirm the hypothesis about the coupled mechanism of osteoclast and osteoblast involvement in bone remodeling during the transition to the stage of reversion and formation.

Osteoclasts stimulate osteoblastogenesis in other ways as well; several factors that produce osteoclasts as well as those released from the matrix during resorption have been isolated and described. Bone resorption by osteoclasts is accompanied by the release of biologically active substances from the matrix (TGF- $\beta$, IGF-1, Afamin, CXC motif ligand 16 (CXCL16), platelet-derived growth factor (PDGF-BB), as well as secretion by osteoclasts of other coupling factors BMP6, SLIT3 (slit guidance ligand 3), component $3 \mathrm{a}(\mathrm{C} 3 \mathrm{a})$, tartrate resistant acid phosphatase (TRAP), cardiotrophin-1 (CT-1), RANK, collagen triple repeat containing1 (CTHCR1), C-X-C motif ligand (CXCL16), PDGF-BB, hepatocyte growth factor (HGF), S1P, which attract mesenchymal stem cells to this area (Kim et al., 2018; Wang et al., 2020).

Afamin secreted by osteoclasts plays an important role in osteoblast genesis. At an early stage of differentiation, Afamin, through the Akt signaling pathway, stimulates the migration of preosteoblasts, promotes delivery of Wnt ligands to their receptors on the cell surface and prevents the aggregation of Wnt proteins (Kim et al., 2012). CT-1 attracts mesenchymal stem cells to this area (Wang et al., 2020). During bone remodeling, osteoclasts stimulate osteoblast chemotaxis while maintaining spatial segregation.

Differentiation of mesenchymal cells into osteoblasts and osteocytes is a multistep process involving transcription factors Sox9, RUNX2, Sp7 and osterix (Osx). The main pathways of osteoblast formation are the 
signaling proteins WNT, BMPs, IGF-1, FGF-2, FGF-18, PDGFs, TGF- $\beta$, ephrin family transmembrane proteins, and, systemic factors - PTH, vitamin D, sex hormones and endogenous GCs (Chen et al., 2012; Siddiqui \& Partridge2016). Excess of GCs, thyroxine, leptin, pyrophosphates, peroxisome proliferator-activated receptor gamma (PPAR $\gamma$ ) and semaphorin-4D (Sema-4D) inhibit osteoblastic differentiation.

Osteoblastic differentiation is enhanced by melatonin, by increasing RUNX2 expression and by inhibiting PPAR $\gamma$, which stimulates adipogenic differentiation of mesenchymal stem cells (Zhang et al., 2010). Also, melatonin restores dexamethasone-induced inhibition of osteoblast differentiation via PI3K / AKT and BMP / Smad signaling pathways (Zhao et al., 2020a).

In bone remodeling at the stage of osteoblastogenesis and formation important signaling pathways are WNT pathways, in which signal transduction by WNT proteins is strictly controlled at several levels. In humans, 19 different Wnt-proteins have been described, which can activate both canonical and non-canonical Wnt-signaling pathways. The canonical Wnt/ $\beta$-catenin signaling pathway is key to mesenchymal cell differentiation, osteoblast activity, and bone formation through regulation of RUNX2 gene expression (Haxaire et al., 2016). This pathway is related to the interaction of Wnt protein- ligands (Wnt 2, 4, 5, 11, 16) with specific transmembrane protein Frizzled (FZD) and its associated low density lipoprotein 5 and 6 co-receptors (LRP-5/6) (Fig. 8).

The activation of this complex is accompanied by an increase in the function of protein Disheveled (DSH), which inhibits the related proteins: glycogen synthase kinase $3 \beta$ (GSK3 $\beta$ ), the adenomatosis polyposis coli (APC), AXIN, casein kinase 1 (CK1) and decreases GSK3 $\beta$ activity by inhibiting phosphorylation, which is accompanied by $\beta$-catenin stabilization and its accumulation in cytoplasm. After translocation into the cell nucleus, $\beta$-catenin interacts with the family of transcription factors, RUNX2, and through family of high mobility group (HMG) stimulates the expression of target genes, including Lef1, Tcf7, Nkd2 and Axin2 (Karner \& Long, 2017). In addition, in osteoblasts, the Wnt / $\beta$-catenin signal transduction pathway controls the expression of $\mathrm{OPG}$, a specific RANKL inhibitor, and thus regulates osteoclastogenesis. Secreted proteins of the Dickkopf family (Dkk) can directly oppose canonical Wnt binding via LRP5 or LRP6 (MacDonald \& He, 2012). Proliferation, differentiation of osteoblasts and suppression of apoptosis are promoted by inhibition of Dickkopf protein (DKK4) expression (Hiramitsu et al., 2013). Sclerostin, a Wnt signal transduction antagonist, was found to be highly expressed in osteocytes, after binding to LRP $5 / 6$ competitively affecting Wnt and LRP 5/6 binding.
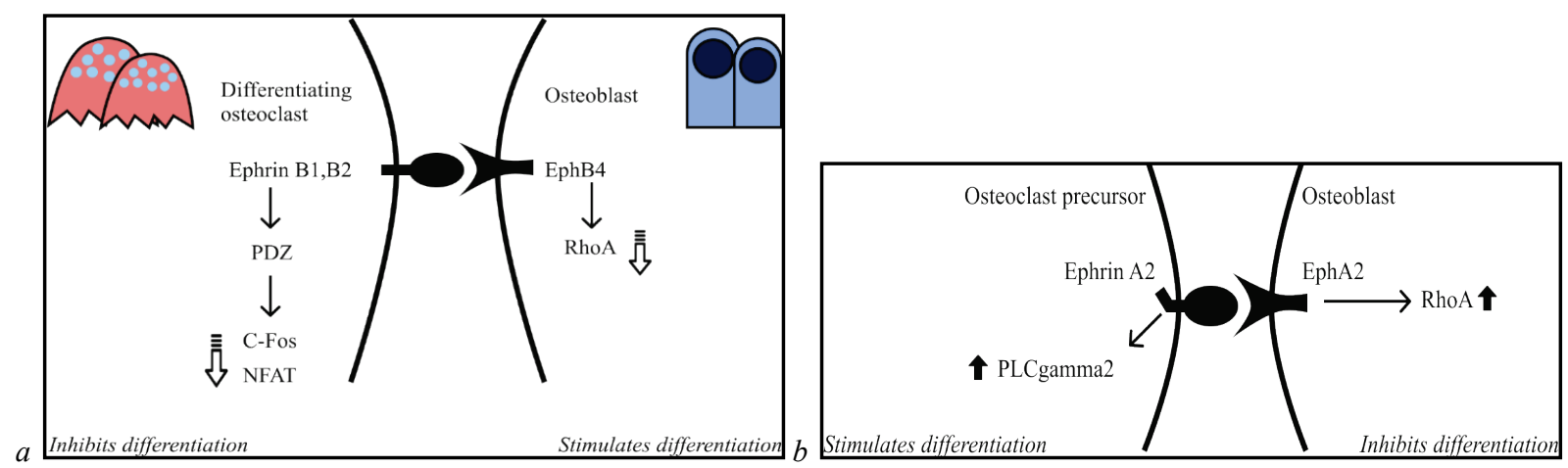

Fig. 7. Signaling pathways (a) EphrinB1, B2 - EphB4; (b) EphrinA2 - EphA2 mutual influence (coupling) of osteoclasts and osteoblasts on proliferation and differentiation (adapted from Matsuo \& Otaki, 2012): ephrin ligand, Eph - ephrin receptor; c-Fos - PDZ - PSD-95/Dlg/ZO-1; NFAT - nuclear factor of activated T cells; PLC $\gamma 2$ - phospholipase C gamma2; RhoA - Ras homolog family member A

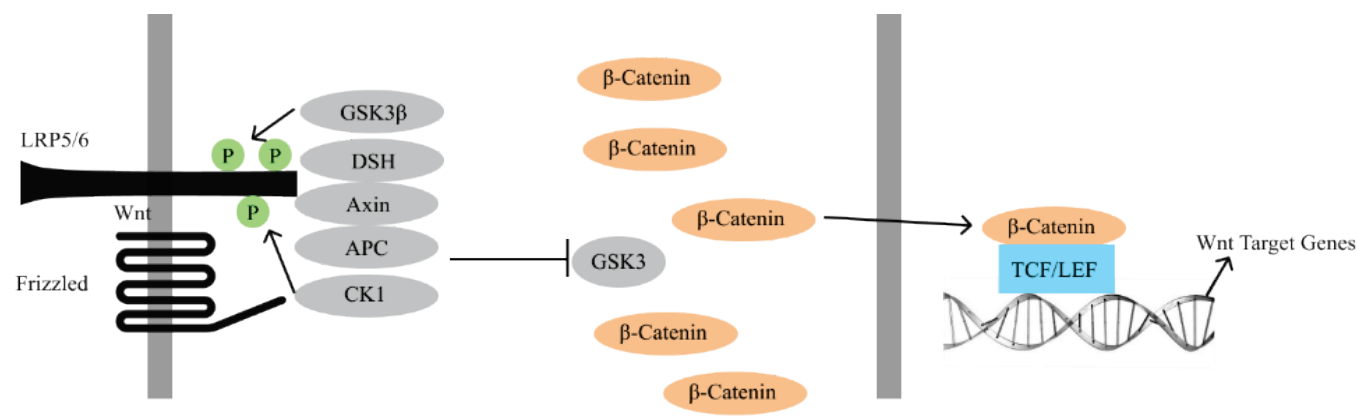

Fig. 8. Signal Wnt-canonical pathway (adapted from Meszaros \& Patocs, 2020): APC-Adenomatosis polyposis coli, LEF-1 - Lymphocyte enhancer-

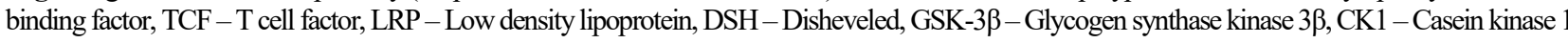

The Wnt signaling pathway also activates signal transduction independent of $\beta$-catenin by activating intracellular cascades involving Gproteins Rho and Rac, calcium-calmodulin-dependent kinase 2 (CaMK2), JNK and p38, phospholipase-C, protein kinase C (PKC), protein kinase A (PKA), PI3K / AKT and mTOR (Karner \& Long, 2017).

Wnt10b induces osteoblastogenesis by activating transcription factors RUNX2, Dlx5 and osterix (Bennett et al., 2005). One of the mechanisms by which Wnt10b promotes osteoblastogenesis is the suppression of the expression of transcription factor CCAAT-enhancer binding protein $(\mathrm{C} / \mathrm{EBP} \alpha)$ and PPAR $\gamma$ that promote the adipogenic commitment of mesenchymal stem cells.

The Wnt-5a ligand of the non-conon signaling pathway inhibits the canonical Wnt pathway, promoting glycogen synthase kinase 3 (GSK-3)independent degradation of $\beta$-catenin (Topol et al., 2003). Wnt5a and
Wnt 5 b promote adipogenesis by increasing PPAR $\gamma$ by activating Hippo signaling Yes-associated protein 1 (YAP) and transcriptional coactivator with TAZ (Ackers \& Malgor, 2018).

Non-canonical Wnt pathways activate signaling independently of $\beta$ catenin, through intracellular cascades involving G-proteins Rho and Rac, calcium calmodulin dependent kinase 2 (CaMK2), c-Jun N-terminal kinase (JNK) and p38, phospholipase-C, PKC, protein kinase A (PKA), PI3K/AKT and mTOR (Karner \& Long, 2017).

BMP-2, transforming growth factor- $\beta$ (TGF-2) and insulin-like growth factor 1 (IGF-1) are involved in the regulation of Wnt signaling pathway that stimulate osteoblastogenesis (Matsuo \& Otaki, 2012; Frenkel et al., 2015; Huntley et al., 2019). IGF-1 affects the stability of $\beta$-catenin and stimulates transcriptional activity. TGF- $\beta$ / BMP signaling plays an important regulatory function in osteoblast differentiation and bone 
formation. Osteoblastogenesis involves cross-talk between TGF- $\beta$ / BMP signaling and many other major signaling pathways such as Wnt, MAPK, Smad, Hedgehog, Notch, and FGF (Chen et al., 2012). BMP-mediated signaling results in increased expression of RUNX2 and osterix, mainly through Smad and MAPK signaling pathways. RUNX2 expression is influenced by the Dix 5 gene, which is activated by BMP-2. The Dix 5 gene protein is considered a key factor in osteoblast maturation (Chen et al., 2012).

RUNX2-dependent transcription is influenced by PTH, BMP, FGF2, mechanical stress transmitted through the matrix (Camilleri \& McDonald, 2006). Post-translational changes act through the signaling pathways MAPK, SMAD4, SMAD1 / 5, and protein kinases A and C (PKA, PKC), which activate RUNX2 by phosphorylation and ubiquination (Franceschi \& Xiao, 2003). BMP-2, BMP-7, and BMP-9 induce osteoblastogenesis, but BMP-9 has a more pronounced effect (Jann et al., 2020). In addition to the Wnt pathway, the role of Notch signaling pathway was identified. In osteoblastic cells, Notch activation suppresses cell differentiation and causes osteopenia of cancellous bone (Zanotti \& Canalis, 2016). Notch can directly interact with RUNX2, by inhibiting Notch function, promotes osteoblast proliferation and differentiation (Infante \& Rodríguez, 2018). Stimulation induces juxta and intramembrane cleavage of Notch proteins; the intracellular Notch domain is released and moves towards the nucleus to regulate gene expression. Notch signaling disrupts RUNX2 activity. Control of target gene expression in osteoprogenitor cells and osteoblasts is also carried out by Wnt and Notch influence on signal transduction to TGF- $\beta$ superfamily (Jann et al., 2020). TGF- $\beta$ promotes apoptosis of osteoclasts and stimulates chemotaxis of preosteoblasts and osteoblasts.

Osterix/Sp7 involvement is necessary for differentiation of preosteoblasts into osteoblasts. This transcription factor stimulates the expression of type I collagen, osteocalcin, osteopontin, sialoprotein and osteonectin genes in cells.

The role of the transcription factor Forkhead box P1 (FOXP1) in the differentiation of mesenchymal cells in the osteogenic direction was established; FOXP1 inhibits adipocyte differentiation by interacting with the key core-binding factor subunit beta $(\mathrm{BF} \beta)$.

The attention of researchers is attracted by small non-coding RNA molecules 18-25 nucleotides of microRNA (miRNAs, MiR), which regulate metabolism by modulating the differentiation and activity of osteoblasts and osteoclasts (Xie et al., 2015; Zuo et al., 2015; Sun et al., 2016; Garcia \& Delany, 2021). So far, more than 20 species of microRNAs affecting the proliferation and differentiation of osteoblasts and 7 species affecting the differentiation of osteoclasts have been isolated. Data from recent studies regarding miRNA in bone cells are summarized in reviews (Garcia \& Delany, 2021). MiR-20a has been shown to upregulate RUNX2, affects TGF $\beta$ isoforms, TGF $\beta R 1$ and TGF $\beta$ R2 signaling receptors, BMP7, BMP2 and BMPR2 receptor, small mothers against decapentaplegic (SMAD), total SMAD4, and inhibits SMAD7 (Garcia \& Delany, 2021). The miR-140 family plays an important role in inhibiting TGF $\beta$ signaling by acting on both ligand and receptor. The miR-106 isoforms inhibit osteoblast differentiation via BMP2 and SMAD5. It was found that MiR-29a cluster by regulating Wnt-3a, glycogen synthase kinase $3 \beta$ and $\beta$-catenin signaling enhances osteoblast differentiation through increased expression of RUNX2 osteocalcin, collagen type 1a1, IGF-1, and also stimulates matrix mineralization (Wang et al., 2013; Lian et al., 2019). Thus, miRNAs are considered to be important modulators of bone remodeling.

Other important factors affecting osteogenesis have been identified. These are long non-coding RNA molecules (LncRNAs), more than 200 nucleotides long, which are involved in many biological processes. Most IncRNAs are located in the nucleus, where they typically regulate gene expression by mediating epigenetic changes such as DNA methylation, histone modification, and chromatin remodeling (Zhao et al., 2020b). The cytoplasm contains small amounts of IncRNAs, where they modulate mRNA stability and translation, and interfere with posttranscriptional regulation (Yoon et al., 2014).

LncRNA-DANCR circulating in monocytes is considered as a potential biomarker of postmenopausal osteoporosis (Tong et al., 2015). A recent published review presents the latest data regarding the role of these molecules in osteoporosis (Zhao et al., 2020b). As shown in this review, many lncRNAs (H19, MALAT1, LOC103691336 and ODSM) can regulate osteogenic differentiation and osteoclastogenesis by modulating gene expression at different levels of transcription, and the likes of lncobl, H19, HOTAIR and PGC1ß-OT1 can directly or indirectly mediate epigenetic changes. Thus, IncRNA HOTTIP interacts with WDR5, a positive regulator of $\beta$-catenin expression, activating Wnt / $\beta$-catenin signal transduction pathway, which promotes osteogenic differentiation of mesenchymal stem cells; in particular, IncNKILA affects the RXFP1/ AKT signal pathway, which is a positive regulator of osteogenesis, LncNKILA overexpression inhibits NF-kB expression and increases RUNX2 expression, which promotes osteogenic differentiation of mesenchymal stromal cells (Zhang et al., 2020). Other IncRNAs have also been described that differentially affect osteogenic differentiation (LncRNA TUG1, MALAT1, IncRNA AK077216, etc.).

The family of semaphorins (Sema), membrane and secreted proteins, plays an important role in the regulation of bone formation. In RANKLactivated osteoclasts, the expression of Sema 4D is increased, which, by binding to the Plexin B1 receptor, inhibits osteoblast differentiation, inhibiting the secretion of alkaline phosphatase and osteocalcin, as well as the formation of mineralized nodules in cell culture (Lontos et al., 2018). In addition, Sema 4D, through its Plexin-B1 receptor, inhibits the locomotion of osteoblasts through contact inhibition of locomotion (CIL) (Deb Roy et al., 2017). Blocking Sema 4D has an anabolic effect on bone.

In contrast to the action of Sema 4D, Sema 3A inhibits osteoclastic bone resorption, enhances bone formation, supports osteocyte viability, and mature osteocyte survival; in an estrogen-dependent manner (Hayashi et al., 2019).

On histological examination, different sections of bone trabeculae may show BMUs at different stages of remodeling: osteoblast-filled formation and resorption with the presence of osteoclasts and the formation of canopies (Fig. 9).

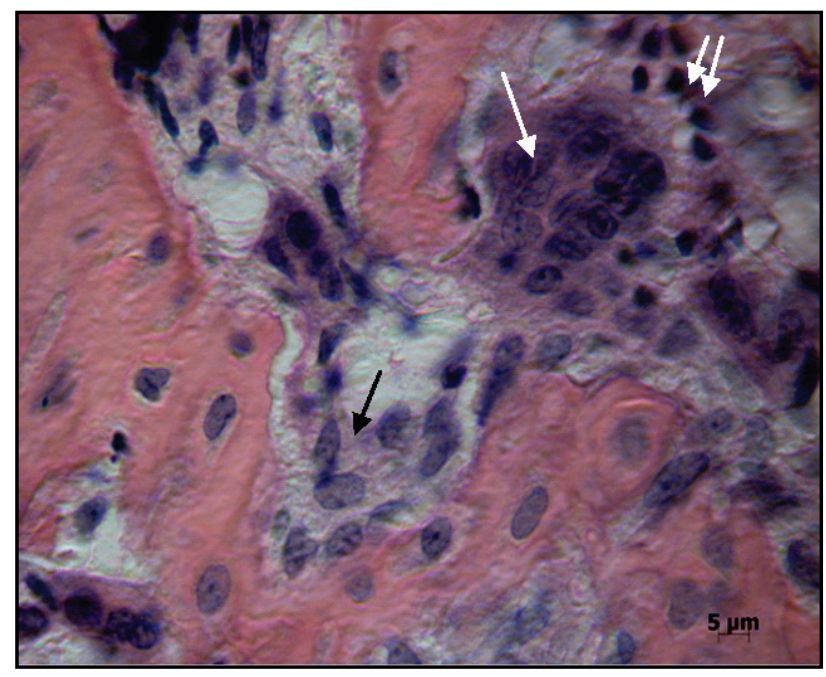

Fig. 9. Bone remodeling: osteoclast in the resorption cavity (white arrow); osteoprogenitor cells form a canopy (double white arrow); osteoblasts in the resorption cavity (black arrow); hematoxylin and eosin

Osteoclasts in the resorptive cavity die by apoptosis after performing their function. Mechanisms of apoptosis through activation of M-CSF - cFMS and TNF- $\alpha-$ TNFR signaling pathways have been described. Binding of M-CSF to its receptor c-FMS through the RAS-dependent mechanism and other transcription factors leads to apoptosis of osteoclasts. In the Ras-dependent mechanism, M-CSF activates PAK1, which promotes osteoclast apoptosis by modulating the expression of survivin, a member of the IAP family (Soysa \& Alles, 2019).

The binding of TNF- $\alpha$ to the receptor TNFR activates TNF receptorassociated death domain (TRADD) TRADD, which interacts with Fasassociated death domain (FADD) through $\mathrm{DD}$, and subsequently promotes the recruitment of caspase 8, which leads to the formation of a signaling complex that induces osteoclast apoptosis (Peng et al., 2018; Soysa \& Alles, 2019) (Fig. 10). 


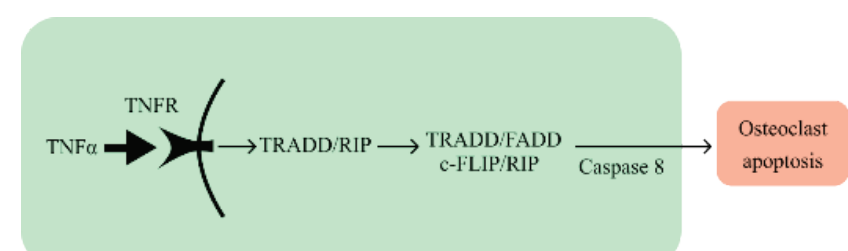

Fig. 10. Osteoclast apoptosis through TNF $\alpha-T N F \beta$ signaling pathway: c-FLIP - Fas-associated death domain-like interleukin-lbeta-converting enzyme-inhibitory protein; FADD - Fas-associated death domain; $\mathrm{TNF} \alpha$ - tumour necrosis factor alpha; TNFR - receptor of tumour necrosis factor; TRADD - TNF receptor-associated death domain; $\mathrm{RIP}$ - receptor-interacting protein

The Fas / FasL system is a powerful mechanism of osteoclast apoptosis. Osteoblasts, by expressing RANKL and M-CSF, up-regulate the Fas receptor in osteoclast progenitors via $\mathrm{NF}-\mathrm{kB}$ and induce Fas-mediated apoptosis (Wu et al., 2005). In mature osteoclasts, other mechanisms of the RANK response function, leading to a reduction in the expression level of Fas and Fas-mediated apoptosis (Wu et al., 2005; Soysa \& Alles, 2019). An increase in the Bcl 2 / Bax ratio and caspase 3 activity may be a potential role of signaling proapoptotic mechanisms.

Osteocytes are considered as the coordinator of bone remodeling during the transition from resorption to formation stage, which, by producing sclerostin, provide the functioning of two opposite mechanisms involved in the process of adaptive bone remodeling (Sapir-Koren \& Livshits, 2014a). Under no load conditions, osteocytes produce high levels of sclerostin, the excess of which disrupts canonical WNT / $\beta$-catenin signaling and activates non-canonical WNT signaling pathways aimed at bone resorption. Under mechanical stress, the secretion of sclerostin is reduced, the WNT / $\beta$-catenin pathway is activated, which stimulates bone formation. Sclerostin acts as a molecular mechanism, regulating the expression of RANKL and OPG.

Formation stage. At this stage, the metabolism of osteoblasts, which produce collagen type 1 , osteocalcin, osteonectin, noncollagen proteins, bone alkaline phosphatase isoenzyme, proteoglycans, and other molecules involved in osteoid formation, becomes more active. In addition, at this stage, the cells secrete vascular endothelial growth factor, which is further involved in the formation of blood vessels in the osteoid (Sims \& Martin, 2014).

Each osteoblast synthesizes the matrix around itself, mineralizes it, mounds it, and turns into an osteocyte. With neighbouring cells, osteocytes are connected by canaliculus, forming a unified network.

The newly formed osteoid mineralizes, a multistep process occurring in the BMU. We consider the primary and secondary phases of mineralization, the primary begins $5-10$ days after the formation of the osteoid, the secondary occurs after the complete filling of the BMU (compact bone or spongy bone) (Boivin et al., 2009). Osteoblasts secrete membrane-bound matrix vesicles with calcium, phosphate and enzymes to digest mineralization inhibitors (such as pyrophosphate and proteoglycans) (Anderson, 2003). Minerals are deposited between collagen fibers. Osteoblasts are surrounded by the mineralized matrix and turn into osteocytes. During the secondary mineralization stage, the mineral component matures, including an increase in the number and size of crystals. Mineralization ends after 90 days in the cancellous bone and after 130 days in the cortical layer of the compact bone (Fernández-Tresguerres-Hernández-Gil et al., 2006).

Osteocytes control osteoid mineralization by expressing phosphate regulating endopeptidase homolog X-linked (PHEX), dentin matrix protein 1 (DMP1) and fibroblastic growth factor 23 (FGF23), which leads to either mineralization of the bone matrix or its inhibition (Sapir-Koren \& Livshits, 2014a; Sapir-Koren \& Livshits, 2014b; Chen et al., 2015). Osteoid mineralization is also regulated by connexin 43 (CX43) / Gap junction alpha-1 protein (GJA1) expression through BMP signaling by osteoclasts that interact with osteoblasts (Shi et al., 2017). BMP-7, which induces the expression of alkaline phosphatase, a marker of osteoblast differentiation, and stimulates bone mineralization.

Other systemic and local factors are involved in mineralization and affect mineral homeostasis in the bones. These include estrogens, androgens, PTH, calcitonin, calcitriol, thyroid hormones, cortisol, growth hor- mone, parathyroid hormone-related protein 1 (PTHrP1) and phosphatonins (secreted frizzled-related protein 4, matrix extracellular phosphoglycoprotein), dentin matrix acid phosphoprotein 1 (DMP1), PHEX, polypeptide N-acetylgalactosammonyl transfer 3(GALNT3), fibroblast growth factor receptor 1 (FGFR1), Klotho, ets.

A new molecular mechanism of vitamin D influence on mineralization was discovered (Jo et al., 2020). Based on the available data that osteoblasts and osteocytes express DKK (Li et al., 2006), the authors showed in an experiment that under the influence of $1,25(\mathrm{OH}) 2 \mathrm{D} 3$ the levels of DKK1 protein expression increase until day 7 after exposure and decrease on the following terms during differentiation of osteoblasts, as their transformation into osteocytes is associated with mineralization. During mineralization, 1,25(OH)2D3 induces DKK expression through $\mathrm{C} / \mathrm{EBP} \beta$ activation.

Terminal stage. At this stage osteoblasts differentiate into osteocytes. Each osteoblast synthesizes a matrix around itself, mineralizes it, becomes engulfed and transforms into an osteocyte. The BMU is filled with bone tissue. With neighbouring cells located in the mineralized matrix, osteocytes are connected by long processes forming a unified network. Osteocytes express sclerostin, which indicates the completion of the remodeling process. Osteocytes play the leading role at this stage, as they secrete inhibitory factors that slow the rate of bone formation, preventing excessive bone formation. In addition, in osteocytes Notch1 signaling leads to the inhibition of bone resorption, which is considered secondary, as a strong factor in suppressing resorption is osteoprotegerin (Zanotti \& Canalis, 2016). In addition, in osteocytes Notch1 can suppress sclerostin biosynthesis with subsequent enhancement of Wnt signaling. A feature of remodeling under normal conditions should be a coupling of bone formation and resorption.

\section{Bone remodeling under the influence of glucocorticoids in excess}

Excess GCs disrupt the functioning of many signaling pathways (Fig. 11) that regulate bone cell proliferation and differentiation. While under physiological conditions significant progress has been made in the study of signaling pathways and GC action on the cells involved in remodeling, under conditions of GC excess, this knowledge is fragmentary.

Resting stage. The peculiarity of this stage is the absence of lining cells on the majority of bone trabeculae, which reflects the negative effect of GCs on bone.

Activation stage. Determination of the bone resorption locus occurs at this stage at sites of osteocyte death. Excess GCs induce osteocyte apoptosis by activation of multiple biomarkers and increased expression of proapoptotic genes by increasing the biosynthesis of caspases 3, 7 and 8, which activate the proapoptotic gene Bim and death receptor Fas / FasL (Espina et al., 2008). Osteocyte apoptosis under the influence of excess GCs can be carried out through a receptor-mediated mechanism through rapid activation of pro-apoptotic kinases, which does not require gene transcription. Pyk2 activation leads to reorganization of the cytoskeleton and is accompanied by loss of cell attachment to the extracellular matrix, and JNK activation causes osteocyte death by increasing reactive oxygen species, which eventually leads to apoptosis or anoikis (Plotkin et al., 2007). There is evidence that osteocytes under low doses of GCs undergo autophagy, whereas high doses of GCs lead to osteocyte apoptosis (Yao et al., 2013). Signaling pathway for autophagy may be the activation of the FAS/CD95 via PTH.

Glucocorticoids cause matrix damage by disrupting branched osteocyte canaliculi, promote the release of paracrine factors that increase local angiogenesis, which increases bone resorption (Goldring, 2015).

In addition, during osteocyte apoptosis, RANKL and proinflammatory cytokines (TNF- $\alpha$ and IL-6) mediated by nuclear protein HMGB1 are released under the influence of GCs, and thus, the products of cell degradation also stimulate osteoclast proliferation and differentiation (Hardy et al., 2018; Wang et al., 2019b). As osteocytes die, the number of possible sites of osteoclast attachment to bone increases.

Resorption stage. Glucocorticoids stimulate mononuclear cell fusion and the formation of multinucleated osteoclasts. However, the influence of molecular pathways of GC on this process has not been studied. Glucocorticoids in excess stimulate RANKL expression and decrease OPG 
expression in osteoblasts and osteocytes, which leads to increased proliferation and differentiation of osteoclasts. The effect of glucocorticoids on RANKL-activated osteoclast progenitor cells leads to increase of their resorptive activity but does not influence the number of formed osteoclasts.

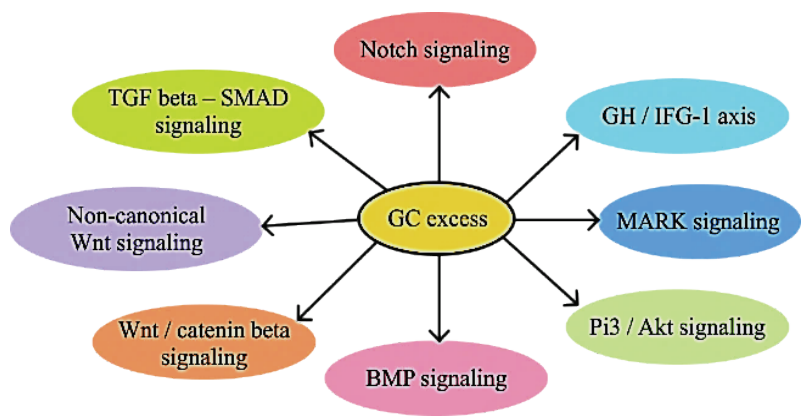

Fig. 11. Effects of glucocorticoids in excess on various signaling pathways in bone remodeling: Akt - kinase, BMP - bone morphogenetic protein, GCs - glucocorticoids, GH - growth hormone, IGF-1 - insulin-like growth factor $1, \mathrm{MAPK}$ - mitogen-activated protein kinases, PI3K phosphoinositide 3-kinase, SMAD - small mothers against decapentaplegic, TGF $\beta$ - transforming growth factor beta, Wnt - wingless and Int-1
Other mechanisms of osteoclastogenesis activation by GCs have been described. One mechanism is related to the action of PTH and D3 in combination with GCs on stromal cells, which leads to an increase in RANKL expression, a decrease in OPG and is accompanied by an increase in the number of osteoclasts (Conaway et al., 2019). Another mechanism of GC influence on the proliferation and activation of osteoclasts through the induction of reactive oxygen species (ROS) is presented. The authors consider this method as a potential therapeutic target in diseases associated with bone mass loss (Shi et al., 2015b).

Glucocorticoids affect osteoclastogenesis in different ways, and the mechanisms of influence are being investigated. By definition (Kim et al., $2006,2007)$ a paradoxical situation develops at this stage. On the one hand, under the influence of GCs osteoclastogenesis is reduced, the motor activity of osteoclasts and their ability to attach to the bone surface is impaired. Inhibition of RhoA, Rac and Vav3 proteins by glucocorticoids is accompanied by disruption of osteoclast cytoskeleton formation and its actin ring providing cell adhesion to bone (Kim et al., 2007). Besides, GCs induce cell apoptosis by transactivation of Bim gene, which leads to expression of apoptotic mediators Bax and Bak. Under their influence the mitochondrial membrane potential is disturbed with subsequent release of cytochrome $\mathrm{C}$ and Apaf-1 into cytosol, which is accompanied by activation of caspase 9 , effector caspases $3,6,7$ and leads to apoptosis (Fig 12).

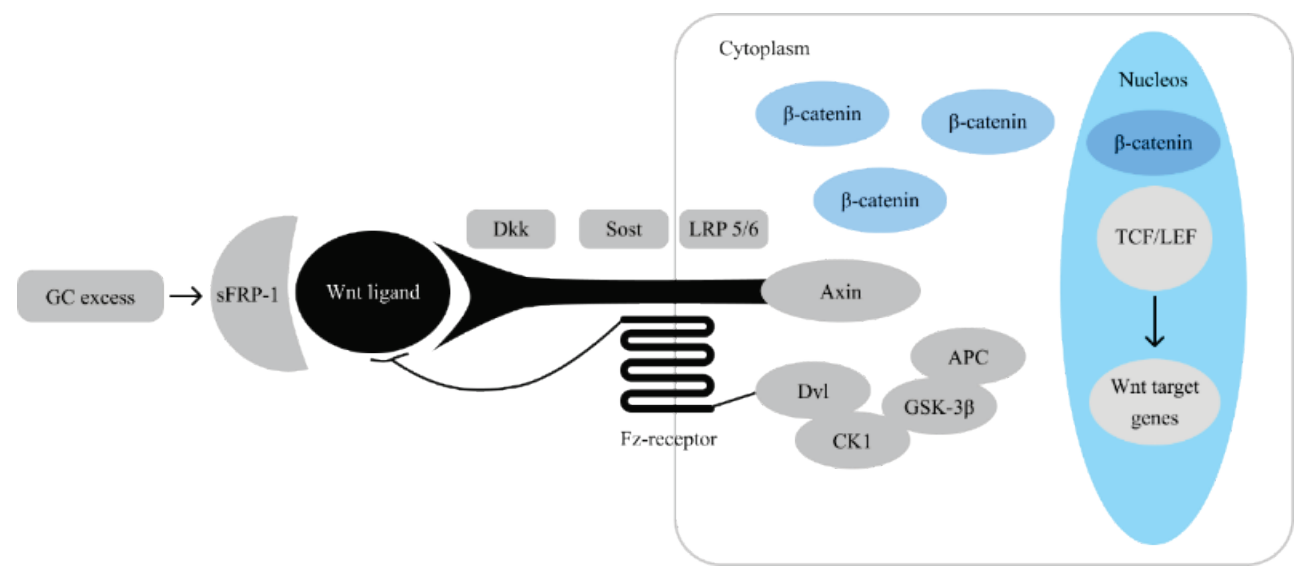

Fig. 12. Excess GCs and the Wnt / $\beta$-catenin signaling pathway (adapted from Meszaros \& Patocs, 2020): APC-adenomatous polyposis coli;

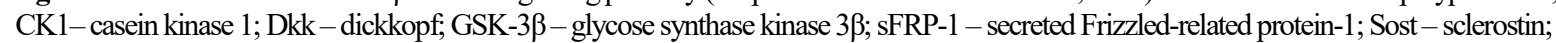
TCF - T-cell factor; LEF - lymphoid enhancer-binding factor; LRP - low-density-lipoprotein-related protein

On the other hand, an increase in the number of osteoclasts and BMU in trabecular and compact bone under the influence of excess GCs is registered. This is associated with an increase in the osteoclast life cycle by inhibition GCs of apoptosis. Excess GCs involving M-CSF - c-FMS signaling pathway through RAS activation leads to the functioning of two independent pathways through Raf and PIK3. In the RANKL - RANK signaling pathway, through cSrc and TRAF6 factors, GCs induce PIK3, AKT and mTOR, which contributes to an increase in the osteoclast life cycle.

Activation of bone resorption by osteoclasts is influenced by increased expression of the cytokine M-CSF, RANKL and IL-6, which stimulate differentiation and proliferation of these cells, against the background of GCs suppression of OPG biosynthesis (Johnson \& Kamel, 2007; Kondo et al., 2008). The dimeric GC receptor present in osteoclasts (contains additional $\beta$-layer) also stimulates RANKL-mediated bone resorption (Conaway et al., 2016). Under the influence of excess GCs, inhibition of caspase 3, the increased level of which causes apoptosis of osteoclasts, which prolongs their viability, has been recorded (Jia et al., 2006, Johnson \& Kamel, 2007; Frenkel et al., 2015).

The peculiarity of early phase therapy with high doses of GCs is the increased formation of osteoclasts, while the number of osteoblasts decreases due to the disruption of molecular interaction in the WNT signaling pathway. However, during treatment, bone resorption slows down, resulting in a state of chronic decreased bone metabolism (Johnson \& Kamel, 2007).

MiR-17 and miR-20a clusters have an inhibitory effect on osteoclastogenesis by reducing the expression of RANKL in osteoblasts. Decrea- sed expression of these microRNAs under the influence of excess GCs enhances bone resorption (Clayton et al., 2018).

The transition from the resorption stage to the next stage of bone formation depends on the RANKL / OPG ratio, which increases under GCs induction, affecting osteoclast activity and changing the bone resorption bone formation balance in the direction of increased resorption. Reduction of OPG under the influence of GCs has a complex multilevel mechanism, a link in which is a direct inhibition of GCs expression of OPG gene (Johnson \& Kamel, 2007; Kondo et al., 2008).

In addition, two GC receptor gene polymorphisms have been found, whose carriers may also differ in their response to GC (Szappanos et al., 2009). Individuals with the Bcll polymorphism (GG genotype) of the GC receptor gene are prone to increased bone resorption under GC therapy, which was found by elevated levels of the serum bone resorption marker $\beta$-CrossLaps and the N-terminal collagen telopeptide type I (NTX) in urine.

While under conditions of normal remodeling the resorption phase ends with programmed death of osteoclasts, which is a counteraction to excessive bone mass loss, under conditions of excess of GCs the life cycle of osteoclasts is increased.

Reversal stage is important in the process of bone remodeling, but in both normal bone and GCO conditions, it is insufficiently studied. There is evidence that in the conditions of GCO the disruption of the interaction between resorption and bone formation occurs precisely at this stage (Jensen et al., 2011; Delaisse, 2014). Histomorphometric analysis of iliac crest biopsies from patients who received GCs for a long time showed that over 
the cavities of osteoclastic resorption in almost the majority of samples no overhangs of osteoprogenitor cells are formed and no bone tissue is formed (Jensen et al., 2015).

The density of osteoprogenitor cells can decrease due to various mechanisms of GC influence on the differentiation of mesenchymal bone marrow progenitor cells into osteoblasts. One way may be GC-influenced differentiation of mesenchymal stromal cells into adipocytes through GC induction of $\mathrm{C} / \mathrm{EBP}$ family transcription factor expression, which activates PPAR $\gamma$ transcription, leading to a reduction in the number of osteoblasts (Han et al., 2019). These data suggest that the provision of functional osteoprogenitor cells deserves attention when seeking strategies to prevent bone mass loss that occurs during remodeling in pathological conditions, including the effects of GCs (Jensen et al., 2015).

Formation stage. Experimental studies have shown that an excess of GCs, especially over a long period of time, disrupts the molecular mechanisms of the Wnt/ $\beta$-catenin signaling pathway from binding to receptor molecules on the cell surface to their nuclear translocation (Guañabens et al., 2014; Chen et al., 2016). Excess of GCs increases the expression of sFRP-1, Dkk-1 and sclerostin inhibitors of Wnt signaling pathway, which affects the proliferation and differentiation of osteoblasts (Meszaros \& Patocs, 2020), affecting WNT signaling cascade proteins (Han et al., 2019). Wnt signaling through LRP 5/6 is inhibited by Dkk-1 (Meszaros \& Patocs, 2020) (Fig. 11). Sclerostin has an inhibitory effect by binding to the extracellular domain of LRP5, and sFRP-1 modulates Wnt signaling by binding to the Wnt ligand and preventing Wnt receptor activation (Meszaros \& Patocs, 2020). GC also decreases phosphorylation of Akt and glycogen synthase-kinase $3 \beta$ (GSK3 $\beta$ ), which enhances $\beta$-catenin degradation (Komori, 2016).

In addition, GCs have an inhibitory effect on the ligands of WNT7B and WNT10 signaling pathways, which are crucial for the proliferation and differentiation of osteoblasts (Mak et al., 2009). These data show the complexity of negative actions in this pathway caused by GCs: disruption of osteoblast proliferation and differentiation, increased apoptosis of osteoblasts and osteocytes, which negatively affects the structural and metabolic parameters of bone (Rizzoli \& Biver, 2015) and leads to osteoporosis (Chen et al., 2014; Briot \& Roux, 2015).

Bone remodeling is regulated by Notch1 and Notch2 signaling pathways. GCs can inhibit osteoblast differentiation by increasing the expression of PPAR $\gamma$ and $\mathrm{C} / \mathrm{EBP} \beta$ in two ways, by stimulating the expression of Notch1 and Notch2 mRNA and by inhibiting the expression of Notch target genes such as Hey1, Hey2, and HeyL in osteoblasts (Han et al., 2019; Zanotti et al., 2019). Synergistic action of Notch and GCs leads to the acquisition of adipocyte phenotype by mesenchymal stem cells.

The molecular target of GCs is BMP-2, which plays a key role in bone formation. BMP-2 activates the expression of RUNX2 through the Dix 5 gene, a key factor of osteoblast maturation (Chen et al., 2012). GCs, through the reduction of BMP-2 activity, inhibit the differentiation of osteoblasts. Glucocorticoid excess inhibits osteoblast proliferation through downregulation of cell cycle activators such as cyclin-dependent kinases (CDK) 2, 4, 6, Cyclin D, oncoprotein c-Myc, and transcription factor 1 (E2F-1), and upregulation of cyclin-dependent kinases p21 (CDKN1A) and p27 (CDKN1B) inhibitors (Komori, 2016), disrupts osteoblast attachment to the matrix, which adversely affects their function (Feng \& McDonald, 2011)

The effect of excess GCs on osteoblasts may be associated with oxidative stress and formation of intracellular ROS, which include free radicals and peroxides (Almeida et al., 2011; Klein, 2015). Oxidative stress leads to impaired osteoblast proliferation, differentiation and apoptosis, which is activated by dexamethasone through the signaling pathway: ROS / PI3K / AKT / GSK3 $\beta$ (Deng et al., 2019). Under oxidative stress, Forkhead Box Protein Os (FOXOs) nuclear transcription factors act as protective molecules by regulating the osteogenic activity of osteoblast precursors by Wnt signaling (Ma et al., 2020). FOXOs stimulate osteoblastogenesis through the $\mathrm{Wnt} / \beta$-catenin signaling pathway.

Dexamethasone-induced impairment of osteoblast differentiation restores melatonin through PI3K / AKT and BMP / Smad signaling pathways (Zhao et al., 2020a).

The role of the transcription hypoxia-inducible factor $1 \alpha(\mathrm{HIF}-1 \alpha)$ was studied. HIF-1 $\alpha$ regulates the expression of the target gene that con- trols cellular metabolism and in bone tissue leads to an increase in osteoblasts, trabecular bone mass and its vascularization (Tseng et al., 2010). Glucocorticoids have been shown to inhibit HIF-1 $\alpha$, resulting in impaired osteoblast metabolism. Amplification of HIF-1 $\alpha$ via the phosphoinositidedependent protein kinase 1 (PDK1) / AKT kinase / mTOR signaling pathway acts as an antagonist that may be useful for treating $\mathrm{GCO}(\mathrm{Xu}$ et al., 2020).

Data from recent studies concerning microRNA in bone cells in GC excess are summarized in a review (Clayton et al., 2015). MicroRNAs can have both positive and negative effects on osteoblastogenesis. In experimental studies on rats it was shown that prolonged GC treatment decreases MiR-29a expression, disrupting Wnt Dkk-1 and Akt (Akt kinase) signaling, and ERK (extracellular-signal-regulated kinase) phosphorylation, which leads to disruption of osteoblast differentiation (Wang et al., 2013). At the same time, there is other evidence that MiR-29a mitigates glucocorticoid induction through its effect on RUNX2 acetylation (Ko et al., 2015). Excess GCs reduce miR-199a-5p, which positively affects WNT, WNT2, and Frizzled4 (FZD4) WNT receptor signaling on the plasma membrane (Shi et al., 2015a). Dexamethasone-inhibited osteogenesis restores miR-216a, promotes osteoblast differentiation, and enhances bone formation by regulating the PI3K / AKT mediated c-Cbl pathway (Li et al., 2015).

Excess GCs modulates miR-34a-5p and miR-199a-5p, which negatively affects the proliferation and differentiation of osteoblastic cells (Komori, 2016). The mechanism of the effect of GCs on microRNA29a was disclosed. Increased doses of glucocorticoids upset the balance between osteogenic and adipogenic differentiation, and microRNA-29a, mitigates GC induction through its effect on RUNX2 acetylation (Ko et al., 2015). In GC-treated mesenchymal stromal cells, miR-204-5p or miR$125 \mathrm{a}-3 \mathrm{p}$ was shown to expand osteogenesis and inhibit adipogenesis by targeting the marker genes PPAR $\gamma$ and $\mathrm{C} / \mathrm{EBP} \alpha$ in gene expression studies of osterix and osteocalcin (Shang et al., 2018).

The role of long non-coding RNAs (lncRNAs) under conditions of GCs in excess has attracted the attention of researchers. Treatment of mesenchymal stromal cells with glucocorticoids promoted upregulation of IncTCONS_00041960, expression of RUNX2, osterix and osteocalcin genes, while expression of adipocyte-specific markers was decreased. In addition, a novel TCONS_00041960-miR-04-5p/miR-125a-3p RUNX2 / GILZ axis was identified which is involved in the regulation of adipogenic and osteogenic differentiation of GC-treated mesenchymal stromal cells (Zhang et al., 2020). It is suggested that long intergenic nonprotein coding RNA 311 (LINC00311) is thought to induce proliferation and inhibit osteoclast apoptosis through inhibition of delta-like ligand 3 (DLL3) expression in the Notch signaling pathway, ultimately demonstrating that LINC00311 and its target gene DLL3 may serve as independent factors that are involved in osteoporosis development (Wang et al., 2018).

The role of long non-coding RNAs (lncRNAs) under conditions of GCs in excess has attracted the attention of researchers. Treatment of mesenchymal stromal cells with GCs, through the regulation of lncTCONS 00041960, promoted an increase in the expression of the RUNX2, osterix, and osteocalcin genes, while the expression of specific markers of adipocytes decreased. In addition, a novel lncTCONS 00041960-miR-04-5p / miR$125 \mathrm{a}-3 \mathrm{p}$ / Runx 2 / GILZ axis was identified that is involved in the regulation of adipogenic and osteogenic differentiation of GC-treated mesenchymal stromal cells (Zhang et al., 2020).

LncRNA MALAT1 has been shown in vitro human osteoblasts to protect cells from dexamethasone-induced damage by activating protein phosphatase $\mathrm{Mg}^{2+} / \mathrm{Mn}^{2+}$-dependent 1e (PPM1E) / 5'-AMP-activated protein kinase (AMPK) signaling (Fan et al., 2018). Another LncRNA1 (EPIC1) also increased the viability of dexamethasone-treated osteoblasts via transcription factor Myc (family of regulator genes) (Zhang et al., 2018).

Studies of the effects of GCs on non-coding small and LncRNA and their role in osteoporosis need further research.

The peculiarity of this stage is the low density of osteoblasts, associated with the induction of apoptosis by excess GCs through genomic, cytoplasmic and caspase cascade signaling (Smith \& Cidlowski, 2010; Mollazadeh et al., 2015). Glucocorticoids regulate gene transcription leucine zipper (GILZ), Bim induction via Fox03A/FKHRL1 transcription 
factor (Smith \& Cidlowski, 2010). Cytoplasmic apoptosis signaling is a multicomponent pathway induced by GCs: decreased mRNA levels of antioxidant enzymes, increased ROS, overexpression of GR species specifically targeting mitochondria, etc. Mitochondria also mediate glucocorticoid-induced cytoplasmic effects, including calcium mobilization, release of reactive oxygen species ROS and ceramide, which promote apoptosis.
This transactivation of the Bim gene leads to the expression of apoptotic mediators Bax and Bak. Under their influence the mitochondrial membrane potential is disrupted with the subsequent release of cytochrome $\mathrm{C}$ and Apaf- 1 into the cytosol, which is accompanied by the activation of caspase 9, effector caspases 3, 6, 7 and leads to apoptosis (Smith \& Cidlowski, 2010) (Fig. 13).

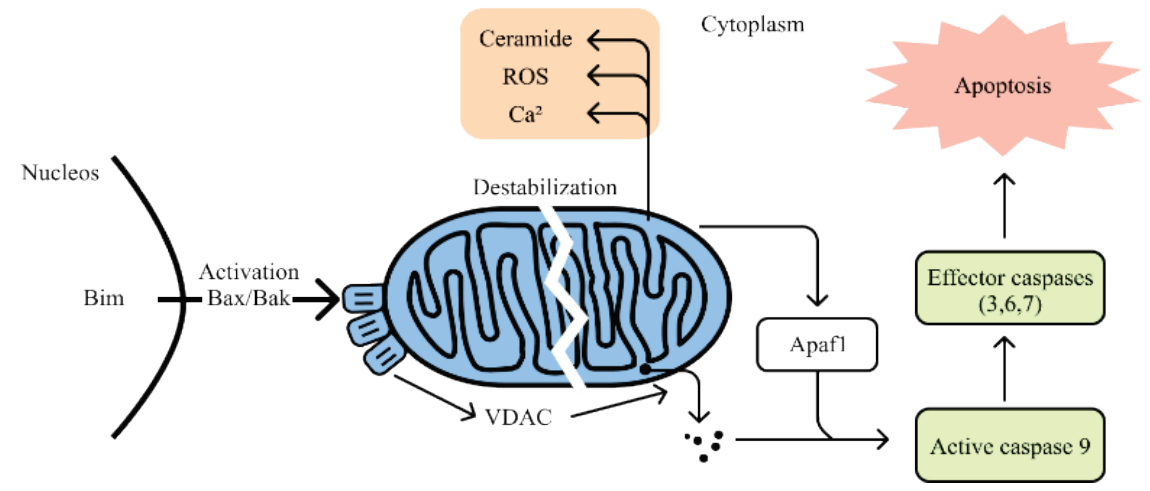

Fig. 13. GC-induced mitochondrial Bim - Bax/Bak signaling disrupts mitochondrial function leading to cell apoptosis (adapted from Smith \& Cidlowski, 2010)

Expression of the key transcription factor of osteoblastogenesis RUNX2 is impaired under the influence of GCs, increases the expression of osterix transcription factor, which inhibits the differentiation and metabolism of osteoblasts and as a consequence decreases the biosynthesis of collagen type 1, proteoglycans, osteocalcin, osteonectin, alkaline phosphatase, $\beta$-glycerophosphate and other proteins involved in osteoid formation by osteoblasts. In addition, GCs inhibit the production of prostaglandin E, IGF-1 and TGF- $\beta$, which activate osteoblasts, which also leads to a decrease in the expression of bone matrix proteins in osteoblasts, leading to disruption of osteoid formation (Ito et al., 2007).

Excess of GCs leads to changes of gene expression in the Wnt signaling pathway, which influences bone formation, and to changes in genes that regulate functional activity of osteoblasts, osteocytes and mineralization of osteoid. That is, already at the stage of osteoid formation, due to a decrease in the pool of osteoblasts, settlement of resorption cavities by osteoblasts and formation of the organic bone matrix is delayed.

Termination stage. Continuous exposure to excess GCs leads to increased expression of DMP1 and Phex genes, which inhibit mineralization. Glucocorticoids in excess stimulate osteocytic osteolysis, which leads to bone matrix destruction, increases the size of osteocyte lacunae, disrupts mineralization by affecting the key biomarkers (Yao et al., 2013; Matsuo, 2014). Due to the predominance of resorption over bone formation, most resorption cavities remain unfilled with bone tissue.

So, excess GCs lead to a disruption of bone remodeling by negatively affecting the major signaling pathways and active molecules involved in this process.

\section{Conclusion}

This review provides a rationale for the cellular and molecular mechanisms of bone remodeling stages under physiological conditions and in glucocorticoid excess. The signaling pathways of cell proliferation, differentiation, viability, and death during remodeling are presented. The main signaling pathways that control bone resorption and formation are RANK / RANKL / OPG, M-CSF - c-FMS, canonical and non-canonical Wnt, Notch, MARK, TGF / SMAD ephrinB1/ephrinB2 - EphB4, TNF $\alpha$ TNF $\beta$, and Bim - Bax/Bak signal pathways. Cytokines, growth factors, prostaglandins, parathyroid hormone, vitamin $\mathrm{D}$, calcitonin, estrogens, non-coding microRNAs and long RNAs also act as regulators of bone remodeling. Excess glucocorticoids negatively affect all stages of bone remodeling, disrupt molecular signaling, induce apoptosis of osteocytes and osteoblasts, increase osteoclast life cycle. Excess glucocorticoids disupt the reversion stage: the formation of canopies of osteoprogenitor cells over resorption cavities, which is critical for the subsequent stages of remodeling. Under the influence of excess glucocorticoids there is a disturbance of osteoid mineralization. Although many signaling pathways involved in resorption and bone formation have been discovered and described, the temporal and spatial mechanisms of their sequential activation and deactivation in cell proliferation and differentiation require additional research.

\section{References}

Ackers, I., \& Malgor, R. (2018). Interrelationship of canonical and non-canonical Wnt signalling pathways in chronic metabolic diseases. Diabetes and Vascular Disease Research, 15(1), 3-13.

Almeida, M., Han, L., Ambrogini, E., Weinstein, R. S., \& Manolagas, S. C. (2011). Glucocorticoids and tumor necrosis factor $\alpha$ increase oxidative stress and suppress Wnt protein signaling in osteoblasts. The Journal of Biological Chemistry, 286(52), 44326-44335.

Amarasekara, D. S., Yun, H., Kim, S., Lee, N., Kim, H., \& Rho, J. (2018). Regulation of osteoclast differentiation by cytokine networks. Immune Network, 18(1), e8

Amiche, M. A., Albaum, J. M., Tadrous, M., Pechlivanoglou, P., Lévesque, L. E., Adachi, J. D., \& Cadarette, S. M. (2016). Fracture risk in oral glucocorticoid users: A Bayesian meta-regression leveraging control arms of osteoporosis clinical trials. Osteoporosis International, 27(5), 1709-1718.

Andersen, T. L., Abdelgawad, M. E., Kristensen, H. B., Hauge, E. M., Rolighed, L., Bollerslev, J., Kjærsgaard-Andersen, P., \& Delaisse, J. M. (2013). Understanding coupling between bone resorption and formation: Are reversal cells the missing link? The American Journal of Pathology, 183(1), 235-246.

Andersen, T. L., Sondergaard, T. E., Skorzynska, K. E., Dagnaes-Hansen, F., Plesner, T. L., Hauge, E. M., Plesner, T., \& Delaisse, J. M. (2009). A physical mechanism for coupling bone resorption and formation in adult human bone. The American Journal of Pathology, 174(1), 239-247.

Anderson, H. C. (2003). Matrix vesicles and calcification. Current Rheumatology Reports, 5, 222-226.

Angeli, A., Guglielmi, G., Dovio, A., Capelli, G., de Feo, D., Giannini, S., Giorgino, R., Moro, L., \& Giustina, A. (2006). High prevalence of asymptomatic vertebral fractures in post-menopausal women receiving chronic glucocorticoid therapy: A cross-sectional outpatient study. Bone, 39(2), 253-259.

Bennett, C. N., Longo, K. A., Wright, W. S., Suva, L. J., Lane, T. F., Hankenson, K. D., \& MacDougald, O. A. (2005). Regulation of osteoblastogenesis and bone mass by Wnt10b. Proceedings of the National Academy of Sciences of the United States of America, 102(9), 3324-3329.

Boivin, G., Farlay, D., Bala, Y., Doublier, A., Meunier, P. J., \& Delmas, P. D. (2009). Influence of remodeling on the mineralization of bone tissue. Osteoporosis International, 20(6), 1023-1026.

Boyce, B. F., \& Xing, L. (2008). Functions of RANKL/RANK/OPG in bone modeling and remodeling. Archives of Biochemistry And Biophysics, 473(2), 139-146.

Boyce, B. F., Li, J., Xing, L., \& Yao, Z. (2018). Bone remodeling and the role of TRAF3 in osteoclastic bone resorption. Frontiers in Immunology, 9, 2263.

Boyle, W. J., Simonet, W. S., \& Lacey, D. L. (2003). Osteoclast differentiation and activation. Nature, 423(6937), 337-342.

Braun, T., \& Zwerina, J. (2011). Positive regulators of osteoclastogenesis and bone resorption in rheumatoid arthritis. Arthritis Research and Therapy, 13, 235. 
Briot, K., \& Roux, C. (2015). Glucocorticoid-induced osteoporosis. RMD Open, 1(1), $\mathrm{e} 000014$

Broege, A., Pham, L., Jensen, E. D., Emery, A., Huang, T. H., Stemig, H., Beppu, M., Petryk, A., O'Connor, M., Mansky, K., \& Gopalakrishnan, R. (2013). Bone morphogenetic proteins signal via SMAD and mitogen-activated protein (MAP) kinase pathways at distinct times during osteoclastogenesis. Journal of Biological Chemistry, 288, 52, 37230-37240.

Buckley, L., Guyatt, G., Fink, H. A., Cannon, M., Grossman, J., Hansen, K. E. Humphrey, M. B., Lane, N. E., Magrey, M., Miller, M., Morrison, L., Rao, M., Robinson, A. B., Saha, S., Wolver, S., Bannuru, R. R., Vaysbrot, E., Osani, M., Turgunbaev, M., Miller, A. S., \& McAlindon, T. (2017). 2017 American College of Rheumatology guideline for the prevention and treatment of glucocorticoid-induced osteoporosis. Arthritis and Rheumatology, 69(8), 1521-1537.

Chen, F., Zhang, L., OuYang, Y., Guan, H., Liu, Q., \& Ni, B. (2014). Glucocorticoid induced osteoblast apoptosis by increasing E4BP4 expression via up-regulation of Bim. Calcified Tissue International, 94(6), 640-647.

Chen, G., Deng, C., \& Li, Y. P. (2012). TGF- $\beta$ and BMP signaling in osteoblast differentiation and bone formation. International Journal of Biological Sciences, $8(2), 272-288$.

Chen, H., Senda, T., \& Kubo, K. Y. (2015). The osteocyte plays multiple roles in bone remodeling and mineral homeostasis. Medical Molecular Morphology, $48(2), 61-68$.

Chen, X., Wang, Z., Duan, N., Zhu, G., Schwarz, E. M., \& Xie, C. (2018). Osteoblast-osteoclast interactions. Connective Tissue Research, 59(2), 99-107.

Chen, Z., Xue, J., Shen, T., Mu, S., \& Fu, Q. (2016). Curcumin alleviates glucocorticoid-induced osteoporosis through the regulation of the Wnt signaling pathway. International Joumal of Molecular Medicine, 37(2), 329-338

Cheon, Y. H., Kim, J. Y., Baek, J. M., Ahn, S. J., Jun, H. Y., Erkhembaatar, M., Kim, M. S., Lee, M. S., \& Oh, J. (2016). WHI-131 promotes osteoblast differentiation and prevents osteoclast formation and resorption in mice. Joumal of Bone and Mineral Research, 31(2), 403-415.

Cherian, K. E., Kapoor, N., \& Paul, T. V. (2017). Glucocorticoid-induced osteoporosis. Indian Journal of Endocrinology and Metabolism, 21(5), 652-654

Cho, S. W. (2015). Role of osteal macrophages in bone metabolism. Journal of Pathology and Translational Medicine, 49(2), 102-104.

Chotiyarnwong, P., \& McCloskey, E. (2020). Pathogenesis of glucocorticoid-induced osteoporosis and options for treatment. Nature Reviews Endocrinology, 16, 437-447.

Chuang, M. H., Chuang, T. L., Koo, M., \& Wang, Y. F. (2017). Trabecular bone score reflects trabecular microarchitecture deterioration and fragility fracture in female adult patients receiving glucocorticoid therapy: A pre-post controlled study. BioMed Research International, 2017, 4210217.

Clayton, S. A., Jones, S. W., Kurowska-Stolarska, M., \& Clark, A. R. (2018). The role of microRNAs in glucocorticoid action. The Journal of Biological Chemistry, 293(6), 1865-1874.

Compston, J. (2018). Glucocorticoid-induced osteoporosis: An update. Endocrine, 61(1), 7-16.

Conaway, H. H., Henning, P., Lie, A., Tuckermann, J., \& Lerner, U. H. (2019). Glucocorticoids employ the monomeric glucocorticoid receptor to potentiate vitamin D3 and parathyroid hormone-induced osteoclastogenesis. FASEB Journal, 33(12), 14394-14409

Conaway, H. H., Henning, P., Lie, A., Tuckermann, J., \& Lerner, U. H. (2016). Activation of dimeric glucocorticoid receptors in osteoclast progenitors potentiates RANKL induced mature osteoclast bone resorbing activity. Bone, 93, 43-54.

Cooper, M. S., Blumsohn, A., Goddard, P. E., Bartlett, W. A., Shackleton, C. H, Eastell, R., Hewison, M., \& Stewart, P. M. (2003). 11beta-hydroxysteroid dehydrogenase type 1 activity predicts the effects of glucocorticoids on bone. Journal of Clinical Endocrinology and Metabolism, 88(8), 3874-3877.

Cruz-Topete, D., \& Cidlowski, J. A. (2015). One hormone, two actions: Anti- and proinflammatory effects of glucocorticoids. Neuroimmunomodulation, 22, 20-32.

Deb Roy, A., Yin, T., Choudhary, S., Rodionov, V., Pilbeam, C. C., \& Wu, Y. I. (2017). Optogenetic activation of Plexin-B1 reveals contact repulsion between osteoclasts and osteoblasts. Nature Communications, $8,15831$.

Delaisse, J. M. (2014). The reversal phase of the bone-remodeling cycle: Cellular prerequisites for coupling resorption and formation. BoneKey Reports, 3, 561 .

Deng, S., Dai, G., Chen, S., Nie, Z., Zhou, J., Fang, H., \& Peng, H. (2019). Dexamethasone induces osteoblast apoptosis through ROS-PI3K/AKT/GSK3 $\beta$ signaling pathway. Biomedicine and Pharmacotherapy, 110, 602-608.

Espina, B., Liang, M., Russell, R. G., \& Hulley, P. A. (2008). Regulation of bim in glucocorticoid-mediated osteoblast apoptosis. Journal of Cellular Physiology, $215(2), 488-496$

Everts, V., Delaissé, J. M., Korper, W., Jansen, D. C., Tigchelaar-Gutter, W., Saftig P., \& Beertsen, W. (2002). The bone lining cell: Its role in cleaning Howship's lacunae and initiating bone formation. Journal of Bone and Mineral Research, 17(1), 77-90

Fan, J. B., Zhang, Y., Liu, W., Zhu, X. H., Xu, D. W., Zhao, J. N., \& Cui, Z. M. (2018). Long non-coding RNA MALAT1 protects human osteoblasts from dexamethasone-induced injury via activation of PPMIE-AMPK signaling. Cellular Physiology and Biochemistry, 51, 31-45.

Feng, X., \& McDonald, J. M. (2011). Disorders of bone remodeling. Annual Review of Pathology, 6, 121-145.

Feng, X., \& Teitelbaum, S. L. (2013). Osteoclasts: New insights. Bone Research, $1(1), 11-26$.

Fenton, C. G., Doig, C. L., Fareed, S., Naylor, A., Morrell, A. P., Addison, O., Wehmeyer, C., Buckley, C. D., Cooper, M. S., Lavery, G. G., Raza, K., \& Hardy, R. S. (2019). 11ß-HSD1 plays a critical role in trabecular bone loss associated with systemic glucocorticoid therapy. Arthritis Research and Therapy, 21(1), 188

Fernández-Tresguerres-Hernández-Gil, I., Alobera-Gracia, M. A., del-Canto-Pingarrón, M., \& Blanco-Jerez, L. (2006). Physiological bases of bone regeneration II. The remodeling process. Medicina Oral, Patologia Oral y Cirugia Bucal, 11(2), E151-E157.

Florencio-Silva, R., Sasso, G. R., Sasso-Cerri, E., Simões, M. J., \& Cerri, P. S. (2015). Biology of bone tissue: Structure, function, and factors that influence bone cells. BioMed Research International, 2015, 421746.

Fong, D., Bisson, M. L., Laberge, G., McManus, S., Grenier, G., Faucheux, N., \& Roux, S. (2013). Bone morphogenetic protein-9 activates Smad and ERK pathways and supports human osteoclast function and survival in vitro. Cellular Signalling, 25(4), 717-728.

Franceschi, R. T., \& Xiao, G. (2003). Regulation of the osteoblast-specific transcription factor, RUNX2: Responsiveness to multiple signal transduction pathways. Journal of Cellular Biochemistry, 88(3), 446-454.

Frenkel, B., White, W., \& Tuckermann, J. (2015). Glucocorticoid-induced osteoporosis. Advances in Experimental Medicine and Biology, 872, 179-215.

Fu, C., \& Shi, R. (2020). Osteoclast biology in bone resorption: A review. STEMedicine, 1(4), e57.

Garcia, J., \& Delany, A. M. (2021). MicroRNAs regulating TGFß and BMP signaling in the osteoblast lineage. Bone, 143, 115791

Goldring, S. R. (2015). The osteocyte: Key player in regulating bone turnover. RMD Open, 1, e000049

Guañabens, N., Gifre, L., \& Peris, P. (2014). The role of Wnt signaling and sclerostin in the pathogenesis of glucocorticoid-induced osteoporosis. Current Osteoporosis Reports, 12, 90-97.

Hachemi, Y., Rapp, A. E., Picke, A. K., Weidinger, G., Ignatius, A., Tuckermann, J. Weidinger, G., Ignatius, A., \& Tuckermann, J. (2018). Molecular mechanisms of glucocorticoids on skeleton and bone regeneration after fracture. Journal of Molecular Endocrinology, 61(1), R75-R90

Han, L., Wang, B., Wang, R., Gong, S., Chen, G., \& Xu, W. (2019). The shift in the balance between osteoblastogenesis and adipogenesis of mesenchymal stem cells mediated by glucocorticoid receptor. Stem Cell Research and Therapy, 10(1), 377

Han, Y., Zhang, L., Xing, Y., Zhang, L., Chen, X., Tang, P., \& Chen, Z. (2018). Autophagy relieves the function inhibition and apoptosis-promoting effects on osteoblast induced by glucocorticoid. International Joumal of Molecular Medicine, $41,800-808$.

Hardy, R. S., Raza, K., \& Cooper, M. S. (2020). Therapeutic glucocorticoids: Mechanisms of actions in rheumatic diseases. Nature Reviews Rheumatology, 16, 133-144.

Hardy, R. S., Zhou, H., Seibel, M. J., \& Cooper, M. S. (2018). Glucocorticoids and bone: Consequences of endogenous and exogenous excess and replacement therapy. Endocrine Reviews, 39(5), 519-548.

Hartmann, K., Koenen, M., Schauer, S., Wittig-Blaich, S., Ahmad, M., Baschant, U., \& Tuckermann, J. P. (2016). Molecular actions of glucocorticoids in cartilage and bone during health, disease, and steroid therapy. Physiological Reviews, 96(2), 409-447.

Haxaire, C., Haÿ, E., \& Geoffroy, V. (2016). Runx2 controls bone resorption through the down-regulation of the Wnt pathway in osteoblasts. The American Journal of Pathology, 186(6), 1598-1609.

Hayashi, M., Nakashima, T., Yoshimura, N., Okamoto, K., Tanaka, S., \& Takayanagi, H. (2019). Autoregulation of osteocyte Sema3A orchestrates estrogen action and counteracts bone aging. Cell Metabolism, 29(3), 627-637.

Hiramitsu, S., Terauchi, M., \& Kubota, T. (2013). The effects of Dickkopf 4 on the proliferation, differentiation, and apoptosis of osteoblasts. Endocrinology, 154(12), 4618-4626.

Huntley, R., Jensen, E., Gopalakrishnan, R., \& Mansky, K. C. (2019). Bone morphogenetic proteins. Their role in regulating osteoclast differentiation. Bone Reports, $10,0207$.

Infante, A., \& Rodríguez, C. I. (2018). Osteogenesis and aging: Lessons from mesenchymal stem cells. Stem Cell Research and Therapy, 9,244.

Ito, S., Suzuki, N., Kato, S., Takahashi, T., \& Takagi, M. (2007). Glucocorticoid induce the differentiation of a mesenchymal progenitor cell line, ROB-C26 into adipocytes and osteoblasts, but fail to induce terminal osteoblast differentiation. Bone, 40(1), 84-92.

Jann, J., Gascon, S., Roux, S., \& Faucheux, N. (2020). Influence of the TGF- $\beta$ Superfamily on osteoclasts/osteoblasts balance in physiological and pathological bone conditions. International Journal of Molecular Sciences, 21(20), 7597. 
Jensen, P. R., Andersen, T. L., Hauge, E. M., Bollerslev, J., \& Delaissé, J. M. (2015). A joined role of canopy and reversal cells in bone remodeling - lessons from glucocorticoid-induced osteoporosis, Bone, 73, 16-23.

Jensen, P. R., Andersen, T. L., Søe, K., Hauge, E. M., Bollerslev, J., Amling, M., Barvencik, F., \& Delaissé, J.-M. (2011). Premature loss of bone remodeling compartment canopies is associated with deficient bone formation: A study of healthy individuals and patients with Cushing's syndrome. Joumal of Bone and Mineral Research, 27(4), 770-780.

Jia, D., O’Brien, C. A., Stewart, S. A., Manolagas, S. C., \& Weinstein, R. S. (2006). Glucocorticoids act directly on osteoclasts to increase their life span and reduce bone density. Endocrinology, 147(12), 5592-5599.

Jo, S., Yoon, S., Lee, S. Y., Kim, S. Y., Park, H., Han, J., Choi, S. H., Han, J. S. Yang, J. H., \& Kim, T. H. (2020). DKK1 induced by 1,25D3 is required for the mineralization of osteoblasts. Cells, 9(1), 236.

Johnson, M. L., \& Kamel, M. A. (2007). The Wnt signaling pathway and bone metabolism. Current Opinion in Rheumatology, 19(4), 376-382.

Kameo, Y., Miya, Y., Hayashi, M., Nakashima, T., \& Adachi, T. (2020). In silico experiments of bone remodeling explore metabolic diseases and their drug treatment. Science Advances, 6(10), eaax0938.

Kanakamedala, A. K., Mahendra, J., Kareem, N., \& Mahendra, L. (2019). Osteoclasts: Multifaceted molecule in vesicular trafficking. Journal of Clinical and Diagnostic Research, 13(8), ZE01-ZE05.

Karner, C. M., \& Long, F. (2017). Wnt signaling and cellular metabolism in osteoblasts. Cellular and Molecular Life Sciences, 74(9), 1649-1657.

Kelly, A., Bowen, H., Jee, Y. K., Mahfiche, N., Soh, C., Lee, T., Hawrylowicz, C., \& Lavender, P. (2008). The glucocorticoid receptor beta isoform can mediate transcriptional repression by recruiting histone deacetylases. The Joumal of Allergy and Clinical Immunology, 121(1), 203-208.

Kenkre, J. S., \& Bassett, J. (2018). The bone remodelling cycle. Annals of Clinical Biochemistry, 5(3), 308-327.

Kim, H. J., Zhao, H., Kitaura, H., Bhattacharyya, S., Brewer, J. A., Muglia, L. J., Ross, P. F., \& Teitelbaum, S. L. (2007). Glucocorticoids and the osteoclast. Annals of the New York Academy of Sciences, 1116, 335-339.

Kim, H. J., Zhao, H., Kitaura, H., Bhattacharyya, S., Brewer, J. A., Muglia, L. J., Ross, F. P., \& Teitelbaum, S. L. (2006). Glucocorticoids suppress bone formation via the osteoclast. Journal of Clinical Investigation, 116(8), 2152-2160.

Kim, J. H., \& Kim, N. (2016). Signaling pathways in osteoclast differentiation. Chonnam Medical Journal, 52(1), 12-17.

Kitaura, H., Kimura, K., Ishida, M., Kohara, H., Yoshimatsu, M., \& Takano-Yamamoto, T. (2013). Immunological reaction in TNF- $\alpha$-mediated osteoclast formation and bone resorption in vitro and in vivo. Clinical and Developmental Immunology, 2013, 181849.

Klein, G. L. (2015). The effect of glucocorticoids on bone and muscle. Osteoporosis and Sarcopenia, 1(1), 39-45.

Ko, J.-Y., Chuang, P.-C., Ke, H.-J., Chen, Y.-S., Sun, Y.-C., \& Wang, F.-S. (2015). MicroRNA-29a mitigates glucocorticoid induction of bone loss and fatty marrow by rescuing Runx 2 acetylation. Bone, $81,80-88$.

Komori, T. (2016). Glucocorticoid signaling and bone biology. Hormone and Metabolic Research, 48, 755-763.

Kondo, T., Kitazawa, R., Yamaguchi, A., \& Kitazawa, S. (2008). Dexamethasone promotes osteoclastogenesis by inhibiting osteoprotegerin through multiple levels. Journal of Cellular Biochemistry, 103(1), 335-345.

La Corte, R., Trotta, F., \& Adami, S. (2010). Glucocorticoid receptors and bone. Current Stem Cell Research and Therapy, 16(32), 3586-3592.

Leightner, A. C., Meyers, C. M. G., Evans, M. D., Mansky, K. C., Gopalakrishnan, R., \& Jensen, E. D. (2020). Regulation of osteoclast differentiation at multiple stages by protein kinase D family kinases. International Journal of Molecular Sciences, 21(1056), 1-20.

Li, H., Li, T., Fan, J., Li, T., Fan, L., Wang, S., Weng, X., Han, Q., \& Zhao, R. C. (2015). miR-216a rescues dexamethasone suppression of osteogenesis, promotes osteoblast differentiation and enhances bone formation, by regulating cCbl-mediated PI3K/AKT pathway. Cell Death and Differentiation, 22(12), 1935-1945.

Li, J., Sarosi, I., Cattley, R. C., Pretorius, J., Asuncion, F., Grisanti, M., Morony, S., Adamu, S., Geng, Z., Qiu, W., Kostenuik, P., Lacey, D. L., Simonet, W. S., Bolon, B., Qian, X., Shalhoub, V., Ominsky, M. S., Zhu Ke, H., Li, X., \& Richards, W. G. (2006). Dkk1-mediated inhibition of Wnt signaling in bone results in osteopenia. Bone, 39(4), 754-766.

Li, Y., Toraldo, G., Li, A., Yang, X., Zhang, H., Qian, W. P., \& Weitzmann, M. N (2007). B cells and T cells are critical for the preservation of bone homeostasis and attainment of peak bone mass in vivo. Blood, 109(9), 3839-3848.

Lian, W. S., Ko, J. Y., Chen, Y. S., Ke, H. J., Hsieh, C. K., Kuo, C. W., Wang, S. Y., Huang, B. W., Tseng, J. G., \& Wang, F. S. (2019). MicroRNA-29a represses osteoclast formation and protects against osteoporosis by regulating PCAFmediated RANKL and CXCL12. Cell Death and Disease, 10(10), 705.

Lin, N. Y., Chen, C. W., Kagwiria, R., Liang, R., Beyer, C., Distler, A., Julia Luther, J., Engelke, K., Schett, G., \& Distler, J. H. W. (2016). Inactivation of autophagy ameliorates glucocorticoid-induced and ovariectomy-induced bone loss. Annals of the Rheumatic Diseases, 75, 1203-1210.

Liu, S., Zhu, W., Li, S., Ma, J., Zhang, H., Li, Z., Zhang, L., Zhang, B., Li, Z., Liang, X., \& Shi, W. (2016). Bovine parathyroid hormone enhances osteoclast bone resorption by modulating V-ATPase through PTH1R. International Journal of Molecular Medicine, 37(2), 284-292.

Lontos, K., Adamik, J., Tsagianni, A., Galson, D. L., Chirgwin, J. M., \& Suvannasankha, A. (2018). The role of semaphorin 4D in bone remodeling and cancer metastasis. Frontiers in Endocrinology, 9, 322.

Ma, X., Su, P., Yin, C., Lin, X., Wang, X., Gao, Y., Patil, S., War, A. R., Qadir, A., Tian, Y., \& Qian, A. (2020). The roles of FOXO transcription factors in regulation of bone cells function. International Joumal of Molecular Sciences, 21(3), 692.

MacDonald, B. T., \& He, X. (2012). Frizzled and LRP5/6 receptors for Wnt/betacatenin signaling. Cold Spring Harbor Perspectives in Biology, 4, a007880.

Mak, W., Shao, X., Dunstan, C. R., Seibel, M. J., \& Zhou, H. (2009). Biphasic glucocorticoid-dependent regulation of Wnt expression and its inhibitors in mature osteoblastic cells. Calcified Tissue International, 85, 538-545.

Mandal, C. C., Das, F., Ganapathy, S., Harris, S. E., Choudhury, G. G., \& GhoshChoudhury, N. (2016). Bone morphogenetic protein-2 (BMP-2) activates NFATc1 transcription factor via an autoregulatory loop involving $\mathrm{Smad} / \mathrm{Akt} / \mathrm{Ca}^{2+}$ signaling. Joumal of Biological Chemistry, 291(3), 1148-1161.

Matsuo, K. (2014) Glucocorticoid and bone. Osteocytic osteolysis: Potential modulation by glucocorticoids. Clinical Calcium, 24(9), 1337-1342.

Matsuo, K. \& Irie, N. (2008). Osteoclast-osteoblast communication. Archives of Biochemistry and Biophysics, 473(2), 201-209.

Matsuo, K., \& Otaki, N. (2012). Bone cell interactions through Eph/ephrin: Bone modeling, remodeling and associated diseases. Cell Adhesion and Migration, 6 , 148-156.

Matsuoka, K. Park, K. A., Ito, M. Ikeda, K, \& Takeshita, S. (2014). Osteoclastderived complement component 3 a stimulates osteoblast differentiation. Joumal of Bone and Mineral Research, 29(7), 1522-1530.

Mazziotti, G., Formenti, A. M., Adler, R. A., Belizikian, J. P., Grossman, A., Sbardella, E., Minisola, S., \& Giustina, A. (2016). Glucocorticoid-induced osteoporosis: Pathophysiological role of GH/IGF-I and PTH/VITAMIN D axes, treatment options and guidelines. Endocrine, 54, 603-611.

Mellis, D. J., Itzstein, C., Helfrich, M. H., \& Crockett, J. C. (2011). The skeleton: A multi-functional complex organ: The role of key signalling pathways in osteoclast differentiation and in bone resorption. The Journal of Endocrinology, 211(2), 131-143.

Meszaros, K., \& Patocs, A. (2020). Glucocorticoids influencing Wnt/ $\beta$-catenin pathway; multiple sites, heterogeneous effects. Molecules, 25(7), 1489.

Mollazadeh, S., Fazly Bazzaz, B. S., \& Kerachian, M. A. (2015). Role of apoptosis in pathogenesis and treatment of bone-related diseases. Joumal of Orthopaedic Surgery and Research, 10, 15.

Morel, A., Blangy, A., \& Vives, V. (2018). Methods to investigate the role of Rho GTPases in osteoclast function. Methods in Molecular Biology, 1821, 219-233.

Nakashima, T., Hayashi, M., Fukunaga, T., Kurata, K., Oh-Hora, M., Feng, J. Q., Bonewald, L. F., Kodama, T., Wutz, A., Wagner, E. F., Penninger, J. M., \& Takayanagi, H. (2011). Evidence for osteocyte regulation of bone homeostasis through RANKL expression. Nature Medicine, 17(10), 1231-1234.

Negishi-Koga, T., \& Takayanagi, H. (2009). $\mathrm{Ca}^{2+}$-NFATc1 signaling is an essential axis of osteoclast differentiation. Immunological Reviews, 231(1), 241-256.

Nicolaides, N. C., Galata, Z., Kino, T., Chrousos, G. P., \& Charmandari, E. (2010). The human glucocorticoid receptor: Molecular basis of biologic function. Steroids, $75(1), 1-12$

Novack, D. V., \& Mbalaviele, G. (2016). Osteoclasts-key players in skeletal health and disease. Microbiology Spectrum, 4(3), 1-31.

Oakley, R. H., \& Cidlowski, J. A. (2013). The biology of the glucocorticoid receptor: New signaling mechanisms in health and disease. The Journal of Allergy and Clinical Immunology, 132(5), 1033-1044.

Omi, M., Kaartinen, V., \& Mishina, Y. (2019). Activin A receptor type 1-mediated $\mathrm{BMP}$ signaling regulates RANKL-induced osteoclastogenesis via canonical SMADsignaling pathway. Joumal of Biological Chemistry, 294, 17818-17836.

Panettieri, R. A., Schaafsma, D., Amrani, Y., Koziol-White, C., Ostrom, R., \& Tliba, O. (2019). Non-genomic effects of glucocorticoids: An updated view. Trends in Pharmacological Sciences, 40(1), $38-49$.

Parfitt, A. (1980). Morphological basis of bone mineral measurements: Transient and steady state effects of treatment in osteoporosis. Mineral and Electrolyte Metabolism, 4, 273-287.

Pathak, J. L., Liu, L., Zhu, Y. Q., \& Bureik, M. (2020). Cytochrome P450 expression patterns in human osteoblasts during osteogenic differentiation with or without TNF $\alpha$ treatment. Biopharmaceutics and Drug Disposition, 41(4-5), 184-191.

Peng, M., Wang, Y., Qiang, L., Xu, Y., Li, C., Li, T., Zhou, X., Xiao, M., \& Wang, J. (2018). Interleukin-35 inhibits TNF- $\alpha$-induced osteoclastogenesis and promotes apoptosis via shifting the activation from TNF receptor-associated death domain (TRADD)-TRAF2 to TRADD-Fas-associated death domain by JAK1/STAT1. Frontiers in Immunology, 9, 1417. 
Pettita, A. R., Changa, M. K., Humeb, D. A., \& Raggatta, L. J. (2008). Osteal macrophages: A new twist on coupling during bone dynamics. Bone, 43(6), 976-978.

Plotkin, L. I., \& Bruzzaniti, A. (2019). Molecular signaling in bone cells: Regulation of cell differentiation and survival. Advances in Protein Chemistry and Structural Biology, 116, 237-281.

Plotkin, L. I., Manolagas, S. C., \& Bellido, T. (2007). Glucocorticoids induce osteocyte apoptosis by blocking focal adhesion kinase-mediated survival. Evidence for inside-out signaling leading to anoikis. The Joumal of Biological Chemistry, 282(33), 24120-24130.

Qi, B., Cong, Q., Li, P., Ma, G., Guo, X., Yeh, J., Xie, M., Schneider, M. D., Liu, H., \& Li, B. (2014). Ablation of Tak1 in osteoclast progenitor leads to defects in skeletal growth and bone remodeling in mice. Scientific Reports, 4, 7158.

Rizzoli, R., \& Biver, E. (2015). Glucocorticoid-induced osteoporosis: Who to treat with what agent? Nature Reviews Rheumatology, 11(2), 98-109.

Ru, J. Y., \& Wang, Y. F. (2020). Osteocyte apoptosis: The roles and key molecular mechanisms in resorption-related bone diseases. Cell Death and Disease, 11(10), 846.

Saag, K. G., Agnusdei, D., Hans, D., Kohlmeier, L. A., Krohn, K. D., Leib, E. S., MacLaughlin, E. J., Alam, J., Simonelli, C., Taylor, K. A., \& Marcus, R. (2016) Trabecular bone score in patients with chronic glucocorticoid therapy-induced osteoporosis treated with alendronate or teriparatide. Arthritis and Rheumatology, 68(9), 2122-2128

Sapir-Koren, R., \& Livshits, G. (2014). Osteocyte control of bone remodeling: Is sclerostin a key molecular coordinator of the balanced bone resorption-formation cycles? Osteoporosis International, 25(12), 2685-2700.

Shang, G., Wang, Y., Xu, Y., Zhang, S., Sun, X., Guan, H., Zhao, X., Wang, Y., Li, Y., \& Zhao, G. (2018). Long non-coding RNA TCONS_00041960 enhances osteogenesis and inhibits adipogenesis of rat bone marrow mesenchymal stem cell by targeting miR-204-5p and miR-125a-3p. Joumal of Cellular Physiology, 233(8), 6041-6051.

Shapiro, I. M., Layfield, R., Lotz, M., Settembre, C., \& Whitehouse, C. (2014). Boning up on autophagy: The role of autophagy in skeletal biology. Autophagy, 10, 7-19.

Shen, G., Ren, H., Shang, Q., Qiu, T., Yu, X., Zhang, Z., Huang, J., Zhao, W., Zhang, Y., Liang, D., \& Jiang, X. (2018). Autophagy as a target for glucocorticoid-induced osteoporosis therapy. Cellular and Molecular Life Sciences, $75,2683-2693$.

Shi, C., Huang, P., Kang, H., Hu, B., Qi, J., Jiang, M., Zhou, H., Guo, L., \& Deng, L (2015a). Glucocorticoid inhibits cell proliferation in differentiating osteoblasts by microRNA-199a targeting of WNT signaling. Joumal of Molecular Endocrinology, 54(3), 325-337.

Shi, C., Zhang, H., Louie, K., Mishina, Y., \& Sun, H. (2017). BMP signaling mediated by BMPR1A in osteoclasts negatively regulates osteoblast mineralization through suppression of Cx43. Journal of Cellular Biochemistry, 118(3), 605-614

Shi, J., Wang, L., Zhang, H., Jie, Q., Li, X., Shi, Q., Huang, Q., Gao, B., Han, Y., Guo, K., Liu, J., Yang, L., \& Luo, Z. (2015b). Glucocorticoids: Dose-related effects on osteoclast formation and function via reactive oxygen species and autophagy. Bone, 79, 222-232.

Siddiqui, J. A., \& Partridge, N. C. (2016). Physiological bone remodeling: Systemic regulation and growth factor involvement. Physiology, 31(3), 233-245.

Sims, N. A., \& Martin, T. J. (2014). Coupling the activities of bone formation and resorption: A multitude of signals within the basic multicellular unit. BoneKEy Reports, 3, 481.

Sinder, B. P., Pettit, A. R., \& McCauley, L. K. (2015). Macrophages: Their emerging roles in bone. Journal of Bone and Mineral Research, 30, 2140-2149.

Smith, L. K., \& Cidlowski, J. A. (2010). Glucocorticoid-induced apoptosis of healthy and malignant lymphocytes. Progress in Brain Research, 182, 1-30.

Søe, K. (2020). Osteoclast fusion: Physiological regulation of multinucleation through heterogeneity-potential implications for drug sensitivity. International Journal of Molecular Sciences, 21(20), 7717.

Soysa, N. S., \& Alles, N. (2019). Positive and negative regulators of osteoclast apoptosis. Bone Reports, 11, 100225.

Sun, M., Zhou, X., Chen, L., Huang, S., Leung, V., Wu, N., Pan, H., Zhen, W., Lu, W., \& Peng, S. (2016). The regulatory roles of microRNAs in bone remodeling and perspectives as biomarkers in osteoporosis. BioMed Research International, 2016, 1652417

Szappanos, A., Patócs, A., Tõke, J., Boyle, B., Sereg, M., Majnik, J., Borgulya, G., Varga, I., Likó, I., Rácz, K., \& Tóth, M. (2009). Bcll polymorphism of the glucocorticoid receptor gene is associated with decreased bone mineral density in patients with endogenous hypercortisolism. Clinical Endocrinology, 71(5), 636-643.

Tasca, A., Astleford, K., Blixt, N. C., Jensen, E. D., Gopalakrishnan, R., Mansky, K. C. (2018) SMAD1/5 signaling in osteoclasts regulates bone formation via coupling factors. PLoS One, 13(9), e0203404.

Teitelbaum, S. L. (2015). Glucocorticoids and the osteoclast. Clinical and Experimental Rheumatology, 33(4, Suppl. 92), S37-S39.

Todosenko, N. M., Koroleva, Y. A., Khaziakhmatova, O. G., Yurova, K. A., \& Litvinova, L. S. (2017). Genomic and non-genomic effects of glucocorticoids [Ge- nomnye i negenomnye effekty glyukokortikoidov]. Genes and Cells, 12(1), $27-$ 33 (in Russian).

Ton, F. N., Gunawardene, S. C., Lee, H., \& Neer, R. M. (2005). Effects of low-dose prednisone on bone metabolism. Joumal of Bone and Mineral Research, 20(3), $464-470$.

Tong, X., Gu, P. C., Xu, S. Z., \& Lin, X. J. (2015). Long non-coding RNA-DANCR in human circulating monocytes: A potential biomarker associated with postmenopausal osteoporosis. Bioscience, Biotechnology, and Biochemistry, 79, 732-737.

Topol, L., Jiang, X., Choi, H., Garrett-Beal, L., Carolan, P. J., \& Yang, Y. (2003). Wnt-5a inhibits the canonical Wnt pathway by promoting GSK-3-independent beta-catenin degradation. The Joumal of Cell Biology, 162(5), 899-908.

Tseng, W. P., Yang, S. N., Lai, C. H., \& Tang, C. H. (2010). Hypoxia induces BMP2 expression via ILK, Akt, mTOR, and HIF-1 pathways in osteoblasts. Joumal of Cellular Physiology, 223(3), 810-818.

Uehara, S., Udagawa, N., \& Kobayashi, Y. (2019). Regulation of osteoclast function via Rho-Pkn3-c-Src pathways. Joumal of Oral Biosciences, 61(3), 135-140.

Varelas, X., Samavarchi-Tehrani, P., Narimatsu, M., Weiss, A., Cockbum, K., Larsen, B. G., Rossant, J., \& Wrana, J. L. (2010). The Crumbs complex couples cell density sensing to Hippo-dependent control of the TGF- $\beta$-SMAD pathway. Developmental Cell, 19(6), 831-844.

Wang, F. S., Chuang, P. C., Lin, C. L., Chen, M. W., Ke, H. J., Chang, Y. H., Chen, Y. S., Wu, S. L., \& Ko, J. Y. (2013). MicroRNA-29a protects against glucocorticoid-induced bone loss and fragility in rats by orchestrating bone acquisition and resorption. Arthritis and Rheumatism, 65(6), 1530-1540.

Wang, H., Yang, G., Xiao, Y., Luo, G., Li, G., \& Li, Z. (2020). Friend or foe? Essential roles of osteoclast in maintaining skeletal health. BioMed Research International, 2020, 4791786

Wang, L., Heckmann, B. L., Yang, X., \& Long, H. (2019a). Osteoblast autophagy in glucocorticoid-induced osteoporosis. Journal of Cellular Physiology, 234(4), 3207-3215.

Wang, T., Yu, X., \& He, C. (2019b). Pro-inflammatory cytokines: Cellular and molecular drug targets for glucocorticoid-induced-osteoporosis via osteocyte. Current Drug Targets, 20(1), 1-15.

Wang, Y., Luo, T. B., Liu, L., \& Cui, Z. Q. (2018). LncRNA LINC00311 Promotes the proliferation and differentiation of osteoclasts in osteoporotic rats through the notch signaling pathway by targeting DLL3. Cellular Physiology and Biochemistry, 47(6), 2291-2306.

Wegler, C., Wikvall, K., \& Norlin, M. (2016). Effects of osteoporosis-inducing drugs on vitamin D-related gene transcription and mineralization in MG-63 and Saos2 cells. Basic and Clinical Pharmacology and Toxicology, 119(5), 436-442.

Weinstein, R. S. (2007). Is long-term glucocorticoid therapy associated with a high prevalence of asymptomatic vertebral fractures? Nature clinical practice. Endocrinology and Metabolism, 3(2), 86-87.

Wu, A. C., Raggatt, L. J., Alexander, K. A., \& Pettit, A. R. (2013). Unraveling macrophage contributions to bone repair. BoneKEy Reports, 2, 373

Wu, X., Pan, G., McKenna, M. A., Zayzafoon, M., Xiong, W. C., \& McDonald, J. M. (2005). RANKL regulates Fas expression and Fas-mediated apoptosis in osteoclasts. Journal of Bone and Mineral Research, 20(1), 107-116.

Xie, J., Guo, J., Kanwal, Z., Wu, M., Lv, X., Ibrahim, N. A., Li, P., Buabeid, M. A., Arafa, E.-S. A., \& Sun, S. (2020). Calcitonin and bone physiology: In vitro, in vivo, and clinical investigations. International Journal of Endocrinology, 2020 3236828.

Xie, Y., Zhang, L., Gao, Y., Ge, W., \& Tang, P. (2015). The multiple roles of microma-223 in regulating bone metabolism. Molecules, 20(10), 19433-19448.

Xu, W. N., Zheng, H. L., Yang, R. Z., Jiang, L. S., \& Jiang, S. D. (2020). HIF-10 regulates glucocorticoid-induced osteoporosis through $\mathrm{PDK} 1 / \mathrm{AKT} / \mathrm{mTOR}$ signaling pathway. Frontiers in Endocrinology, 10, 922.

Yamakawa, T., Okamatsu, N., Ishikawa K., Kiyohara, S., Handa, K., Hayashi, E. Sakai, N., Karakawa, A., Chatani, M., Tsuji, M., Inagaki, K., Kiuchi, Y., Negishi-Koga, T., \& Takami, M. (2020). Novel gene Merlot inhibits differentiation and promotes apoptosis of osteoclasts, Bone, 2020, 115494

Yao, W., Dai, W., Jiang, J. X., \& Lane, N. E. (2013). Glucocorticoids and osteocyte autophagy. Bone, 54(2), 279-284

Yoon, J. H., Abdelmohsen, K., \& Gorospe, M. (2014). Functional interactions among microRNAs and long noncoding RNAs. Seminars in Cell and Developmental Biology, 34, 9-14.

Zanotti, S., \& Canalis, E. (2016). Notch signaling and the skeleton. Endocrine Reviews, 37(3), 223-253.

Zanotti, S., Yu, J., Adhikari, S., \& Canalis, E. (2018). Glucocorticoids inhibit notch target gene expression in osteoblasts. Joumal of Cellular Biochemistry, 119(7), 6016-6023.

Zarei, A., Morovat, A., Javaid, K., \& Brown, C. P. (2016). Vitamin D receptor expression in human bone tissue and dose-dependent activation in resorbing osteoclasts. Bone Research, 4, 16030.

Zayny, A., Almokhtar, M., Wikvall, K., Ljunggren, Ö., Ubhayasekera, K., Bergquist, J., Kibar, P., \& Norlin, M. (2019). Effects of glucocorticoids on vitamin D3- 
metabolizing 24-hydroxylase (CYP24A1) in Saos-2 cells and primary human osteoblasts. Molecular and Cellular Endocrinology, 496, 110525.

Zhang, L., Su, P., Xu, C., Chen, C., Liang, A., Du, K., Peng, Y., \& Huang, D. (2010) Melatonin inhibits adipogenesis and enhances osteogenesis of human mesenchymal stem cells by suppressing PPAR $\gamma$ expression and enhancing Runx 2 expression. Joumal of Pineal Research, 49(4), 364-372.

Zhang, X. Y., Shan, H. J., Zhang, P., She, C., \& Zhou, X. Z. (2018). LncRNA EPIC1 protects human osteoblasts from dexamethasone-induced cell death. Biochemical and Biophysical Research Communications, 503(4), 2255-2262.

Zhang, Y., Cao, X., Li, P., Fan, Y., Zhang, L., Ma, X., Sun, R., Liu, Y., \& Li, W. (2020). LncRNA NKILA integrates RXFP1/AKT and NF- $\kappa B$ signalling to regulate osteogenesis of mesenchymal stem cells. Joumal of Cellular and Molecular Medicine, 24(1), 521-529.
Zhao, R., Tao, L., Qiu, S., Shen, L., Tian, Y., Gong, Z., Tao, B. Z., \& Zhu, Y. (2020a). Melatonin rescues glucocorticoid-induced inhibition of osteoblast differentiation in MC3T3-E1 cells via the PI3K/AKT and BMP/Smad signalling pathways. Life Sciences, 118044.

Zhao, W., Wang, G., Zhou, C., \& Zhao, Q. (2020). The regulatory roles of long noncoding RNAs in osteoporosis. American Journal of Translational Research, 12(9), 5882-5907.

Zuo, B., Zhu, J., Li, J., Wang, C., Zhao, X., Cai, G., Li, Z., Peng, J., Wang, P., Shen, C., Huang, Y., Xu, J., Zhang, X., \& Chen, X. (2015). microRNA-103a functions as a mechanosensitive microRNA to inhibit bone formation through targeting Runx2. Journal of Bone and Mineral Research, 30(2), 330-345. 